\title{
ROSES RUSTIQUES ET LEUR CULTURE AU CANADA
}

\author{
Par W. T. MACOUN, \\ HORTICULTEUR DU DOMINION \\ ET
}

ISABELLA PRESTON

SPECIALISTE EN HORTICULTURE ORNEMENTALE

\section{DOMINION DU CANADA}

MINISTÈRE FÉDÉRAL DE L'AGRICULTURE

BULLETIN NO 17-NOUVELLE SÉRIE

\section{SERVICE DE L'HORTICULTURE \\ FERMES EXPÉRIMENTALES FÉDÉRALES}

Tradxil ax Bureau de traduction du Ministere

fr.

c. 2 lié par ordre de l'Hon. W. R. Motherwell, Ministre de l'Agriculture,
Ottawa, 1923 


\title{
DIVISION DES FERMES EXPÉRIMENTALES FÉDÉRALES
}

\section{PERSONNEL}

\author{
Directeur, E. S. Archibald, B.A., B.S.A.
}

Eleveur du Dominion.. . . . . . . . . . . . . . . . . G. B. Rothwell, B.S.A.

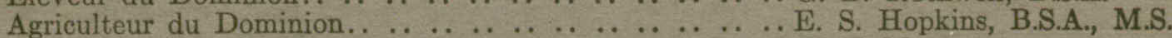

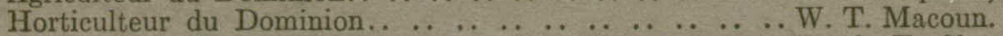

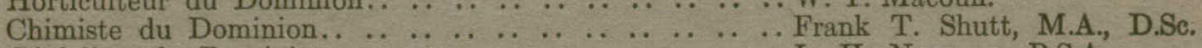

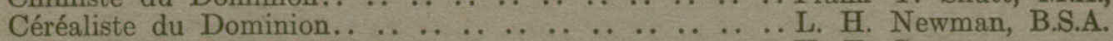

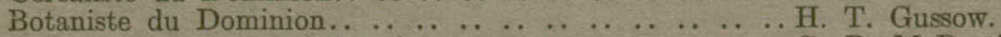

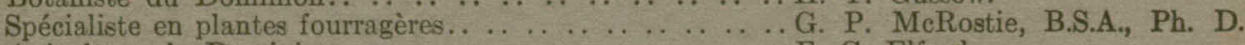

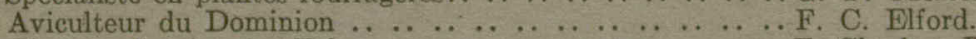

Chef du Service des Tabaes..

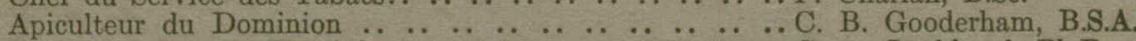

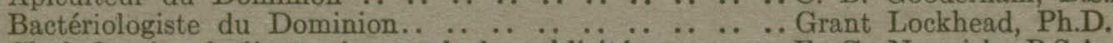

Chef, Service de l'extension et de la publicité.. ... ... . . F. C. Nunnick, B.S.A.

Surveillant en chef des stations de démonstration....... John Fixter.

Spécialiste en fibres économiques.............. . . . . Hutchinson.

\section{ALBERTA}

Régisseur, station expérimentale, Lacombe, Alta., F. H. Reed, B.S.A.

Régisseur, station expérimentale, Lethbridge, Alta, W. H. Fairfield, M.Sc.

Régisseur, sous-station expérimentale, Beaverlodge, Alta, W. D. Albright.

Régisseur, sous-station expérimentale, Fort Vermilion, Alta., Robt. Jones.

\section{COLOMBIE-BRITANNIQUE}

Régisseur, ferme expérimentale, Agassiz, C.-B., W. H. Hicks, B.S.A.

Régisseur, station expérimentale, Summerland, C.-B,, R. H. Helmer.

Régisseur, station expérimentale, Invermere, C.-B., R. G. Newton, B.S.A.

Régisseur, station expérimentale, Sidney, C.-B., E. M. Straight, B.S.A.

\section{MANITOBA}

Régisseur, ferme expérimentale, Brandon, Man., W. C. McKillican, B.S.A.

Régisseur, station expérimentale, Morden, Man., W. R. Leslie, B.S.A.

\section{SASKATCHEWAN}

Régisseur, ferme expérimentale, Indian-Head, Sask., N. D. MeKenzie, B.S.A.

Régisseur, station expérimentale, Rosthern, Sask., W. A. Munro, B.A., B.S.A.

Régisseur, station expérimentale, Scott, Sask., M. J. Tinline, B.S.A.

Régisseur, station expérimentale, Swift-Current, Sask., T. G. Taggart, B.S.A.

\section{NOUVEAU-BRUNSWICK}

Régisseur, station expérimentale, Fredericton, N.-B., C. F. Bailey, B.S.A.

\section{NOUVELLE-ÉCOSSE}

Régisseur, ferme expérimentale, Nappan, N.-E., W. W. Baird, B.S.A.

Régisseur, station expérimentale, Kentville, N.-E., W. S. Blair.

\section{ÎLE DU PRINCE-ÉDOUARD}

Régisseur, station expérimentale, Charlottetown, I. P.-E., J. A. Clark, B.S.A.

\section{ONTARIO}

Ferme expérimentale centrale, Ottawa, Ont.

Régisseur, station expérimentale, Kapuskasing, Ont., S. Ballantyne.

Régisseur, station expérimentale à tabac, Harrow, Ont., D. D. Digges, B.S.A., M.S.A.

\section{QUÉBEC}

Régisseur, station expérimentale, Cap Rouge, Qué., G. A. Langelier, D.Sc.A.

Régisseur, station expérimentale, Lennoxville, Qué., J. A. McClary.

Régisseur, station expérimentale, Ste-Anne de la Pocatière, Qué., J. A. Ste Marie, B.S.A.

Régisseur, station expérimentale, La Ferme, Qué., P. Fortier, Agr.

Régisseur, station expérimentale à tabac, Farnham, Qué., J. E. Montreuil, B.S.A. 
Introduction 3

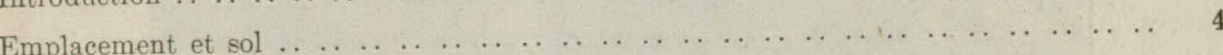

Plantes et plantation $\ldots \ldots \ldots \ldots \ldots \ldots$

Culture, arrosage et fumure $\ldots \ldots \ldots \ldots \ldots \ldots \ldots \ldots \ldots \ldots \ldots \ldots$

Taille $\ldots \ldots \ldots \ldots \ldots \ldots \ldots \ldots \ldots \ldots \ldots$

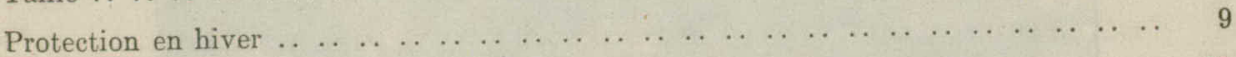

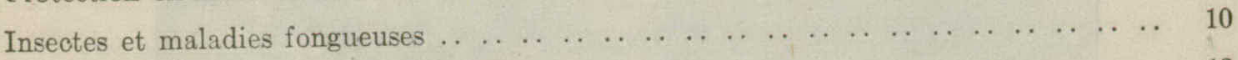

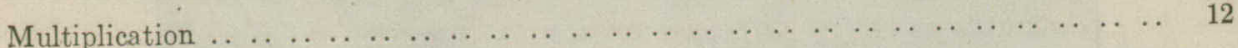

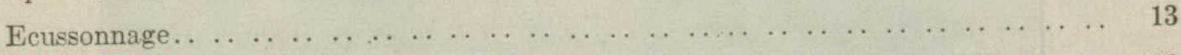

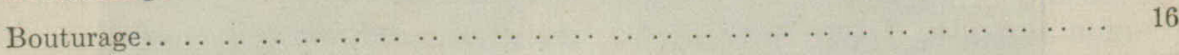

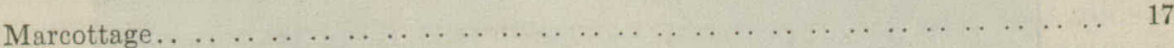

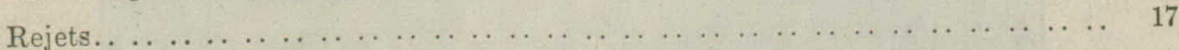

Production de nouvelles variétés. . . . . . . . . . . . . . . . . . 17

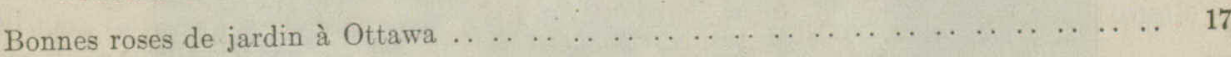

Bonnes roses thé hybrides pour le jardin $\ldots \ldots \ldots \ldots \ldots \ldots \ldots \ldots \ldots \ldots \ldots \ldots \ldots \ldots 18$

Nouvelles roses thé hybrides $\ldots \ldots \ldots \ldots \ldots \ldots \ldots \ldots \ldots \ldots \ldots \ldots \ldots \ldots \ldots \ldots \ldots 18$

Bonnes roses hybrides remontantes pour le jardin $\ldots \ldots \ldots \ldots \ldots \ldots \ldots \ldots \ldots \ldots \ldots$. . . 18

Collection spéciale de roses rustiques pour un petit jardin $\ldots \ldots \ldots \ldots \ldots \ldots \ldots \ldots \ldots$

Roses pernetianas.. . . . . . . . . . . . . . . . . . . . . . . . . . . . . 22

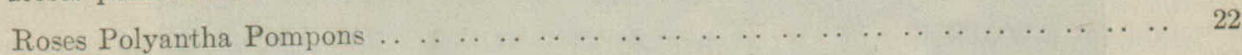

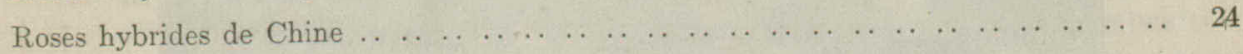

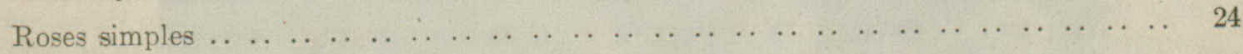

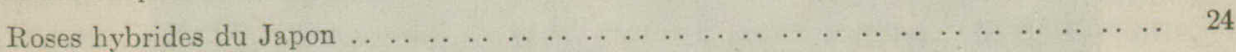

Diverses roses rustiques à $\operatorname{Ottawa} \ldots \ldots \ldots \ldots \ldots \ldots \ldots \ldots \ldots \ldots \ldots \ldots \ldots \ldots \ldots \ldots \ldots \quad \ldots \ldots$

Roses fleurissant tard en automne $\ldots \ldots \ldots \ldots \ldots \ldots \ldots \ldots \ldots \ldots \ldots \ldots \ldots \ldots \ldots . . \ldots 25$

Roses remontantes - Liste des variétés les meilleures et les plus rutiques ........ $\ldots 25$

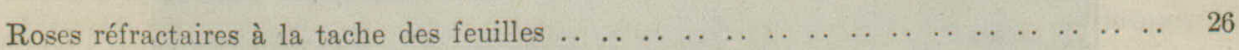

Notes sur l'origine d'espèces populaires de roses $\ldots \ldots \ldots \ldots \ldots \ldots \ldots \ldots \ldots \ldots \ldots \ldots 26$

Hybrides remontantes. . . . . . . . . . . . . . . . . . . . . . . . . . . . . 26

Hybrides Thé.$\ldots \ldots \ldots \ldots \ldots \ldots \ldots \ldots \ldots \ldots \ldots \ldots \ldots \ldots \ldots \ldots \ldots \ldots \ldots{ }_{2} \ldots \ldots \ldots$

Pernetianas. . . . . . . . . . . . . . . . . . . . . . . . . . 27

Notes sur les parents de quelques roses modernes.. . . . . . . . . . . . . . 28

Bonnes roses pour les régionśs de la côte de la Colombie britannique . . . . . . . . . 28

$\mathrm{La}$ rose idéale de jardin $\ldots \ldots \ldots \ldots \ldots \ldots \ldots \ldots \ldots \ldots \ldots \ldots \ldots \ldots \ldots \ldots \ldots \ldots \ldots \ldots \ldots \ldots{ }^{29}$

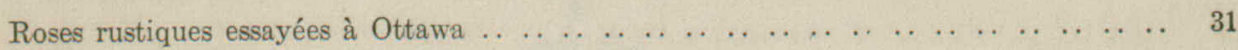




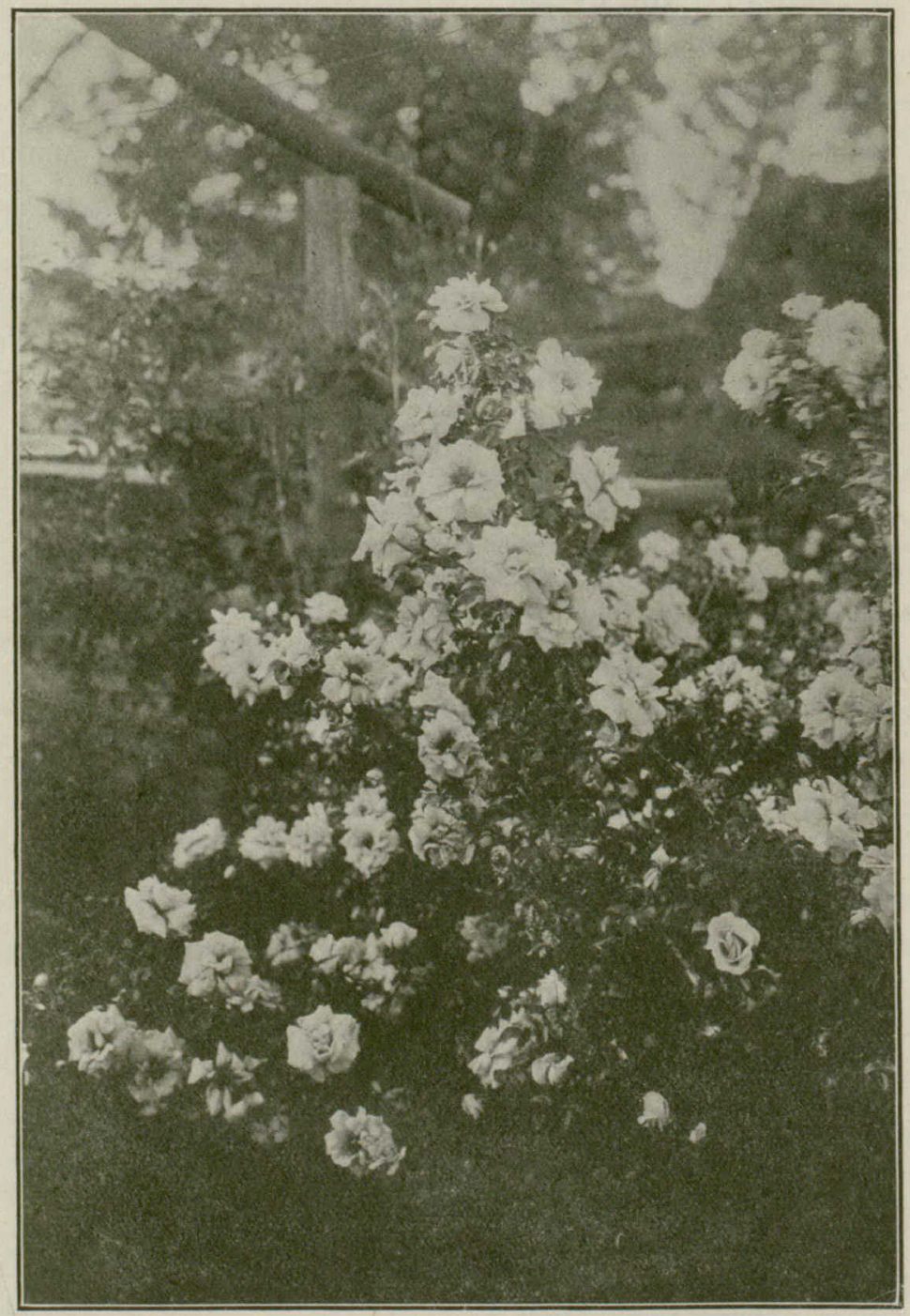

\section{ROSE AGNES .}

Rugosa x Jaune de Perse. Produite à la ferme expérimentale centrale, Ottawa, par feu le docteur Wm. Saunders. 


\title{
ROSES RUSTIQUES
}

\section{ET LEUR CULTURE AU CANADA}

\author{
PAR
}

W. T. MACOUN, horticulteur du Dominion,

\section{ISABELLA PRESTON, spécialiste en horticulture ornementale}

De tout temps, la rose a eu la réputation d'être la plus belle des fleurs, On l'appelle souvent la "reine des fleurs" et elle mérite ce beau titre. Sa beauté a été chantée par les poètes de tous les siècles, et sa culture a fait l'objet de nombreux volumes. Elles nous vient, sous sa forme améliorée, du lointain Orient mais elle se rencontre aujourd'hui dans presque tous les jardins. Son parfum exquis, sa forme gracieuse, les couleurs attrayantes que déploient ses nombreuses variétés, constituent un rare ensemble de qualités; et si l'on y ajoute la beauté du feuillage, la longueur de la tige et la longue durée de la saison de floraison, quelle autre fleur peut soutenir la comparaison avec la rose?

La roseraie est un des endroits les plus agréables que l'on puisse trouver. Malheureusement les jardins de roses sont relativement rares au Canada. La plupart des personnes qui aiment les fleurs désirent cultiver les roses, mais beaucoup de celles qui ont planté quelques rosiers n'ont pas réussi et ont perdu courage.

Nous espérons que les échecs seront moins nombreux à l'avenir, si l'on suit. les conseils de ce bulletin. Certains rosiers sont, il est vrai, plus difficiles à cultiver que la plupart des autres arbrisseaux d'ornement mais il en est cependant dont la culture est très facile; ceux qui ne sont pas prêts à donner à l'hybride remontante, à la rose thé hybride et aux autres roses thé toute l'attention que mérite leur beauté, trouveront dans les hybrides Rugosa, les roses églantines d'Autriche, de Provence et de Damas, un très bel assortiment de roses rustiques, de culture facile, qui n'exigent que peu de taille et ne sont que peu sujettes aux attaques des insectes ou des champignons.

$\mathrm{Au}$ point de vue de la rusticité, les roses généralement cultivées au Canada peuvent se diviser en quatre groupes.

Groupe le plus rustique.-Rosa Rugosa et ses hybrides, roses autrichiennes et de Provence, roses de Damas et roses mousseuses.

Roses du deuxième degré de rusticité.-Hybrides perpétuelles ou remontantes, roses grimpantes du groupe multiflore et roses naines Polyantha. tiana.

Roses du troisième degré de rusticité.-Roses hybrides de thé, roses Perne-

Roses du quatrième degré de rusticité.-Roses thé.

Il y a de grandes différences de rusticité entre les variétés de divers groupes.

Les roses du premier groupe n'exigent que peu ou point de protection dans certaines parties du Canada; les autres doivent être protégées. 
EMPLACEMENT ET SOL

Les roses exigent une lumière abondante; il faudra donc choisir de préférence un endroit qui reçoive le soleil la plus grande partie du jour. A défaut d'un endroit de ce genre le meilleur emplacement est celui qui reçoit le soleil du matin mais il faut éviter tout endroit placé près d'un mur ou d'un bâtiment et qui recevrait par réflection la chaleur du milieu du jour et de l'après-midi, car la plupart des plantes et des fleurs souffrent de cette chaleur brûlante. L'exposition au sud ou au sud-est est préférable. Comme les roses exigent une grande quantité d'humidité, la plantation ne doit pas être à proximité de racines d'arbres qui enlèvent au sol beaucoup d'eau et beaucoup de nourriture. Sur la prairie il est bon d'éviter l'exposition aux vents et de rechercher les endroits où la neige s'amoncelle. Ces deux règles s'appliquent également à toutes les parties du Canada. On apportera d'autant plus de soin au choix du terrain sur lequel la plantation

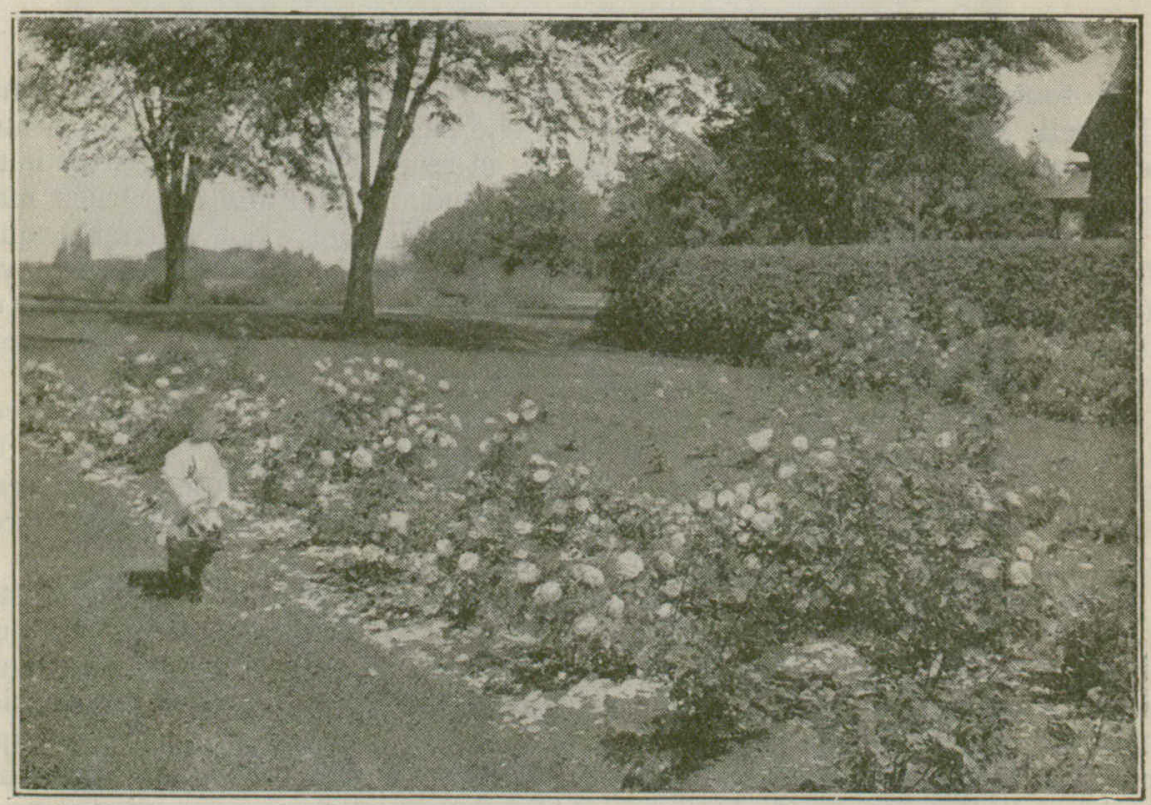

Innocence et beauté. A la roseraie de la ferme expérimenta'e d'Ottawa.

doit être établie que les roses sont plus ou moins résistantes à l'hiver. Les roses du groupe le plus rustique viennent bien sur une grande variété de sols. Les roses des autres groupes sont plus capricieuses, car si les hybrides remontantes viennent mieux dans une terre argilo-sableuse fraîche, mais bien égouttée, les roses thé exigent un sol plus chaud, sablo-argileux de préférence. Cependant, chaque fois qu'il est possible de le faire, on peut choisir un sol intermédiaire qui convienne à tous les groupes. En Angleterre les roses viennent beaucoup mieux que dans la plupart des parties du Canada, l'air plus humide, le sol plus frais, leur sont plus propices que l'air sec et le sol chaud dans bien des parties de notre pays. Il faut done s'attacher à choisir un sol naturellement frais; les terrains de ce genre renferment généralement beaucoup d'humus et retiennent bien l'humidité. Les sols argileux sont généralement plus riches que les sols sablonneux, et comme les roses exigent beaucoup de nourriture, les premiers ont done l'avantage sous ce rapport. *Il faut éviter les sols minces, exposés à devenir très secs et très chauds pendant une sécheresse, conditions nuisibles à la croissance des rosiers. 
Les sols où le niveau d'eau est à moins de trois pieds de la surface sont également à condamner; ils sont froids et les roses n'y fleurissent pas bien. On voit souvent dans ces sols des rosiers qui se développent très bien mais qui ne fleurissent pas, ce qui surprend beaucoup l'amateur. Le sol doit être frais mais bien égoutté, profond et riche; s'il ne l'est pas naturellement et que l'on n'ait pas le choix, on doit le rendre aussi parfait que possible sous ce rapport. On doit toujours appliquer une forte couche de fumier, même sur un sol naturellement riche, et on incorpore ce fumier parfaitement avant de planter les rosiers. Pour préparer un bon terrain, sur un sol qui n'est pas naturellement profond et qui est mal égoutté, enlever d'abord la bonne terre de la surface, puis le sous-sol jusqu'à ce que l'on ait enlevé au total une couche de terre d'environ deux pieds d'épaisseur. Puis, si le sol a besoin de drainage, poser des tuyaux de trois pouces au fond, avec une pente suffisante pour enlever l'eau de surplus qui, naturellement, doit avoir un débouché quelque part. Si ce mode de drainage est impossible, mettre

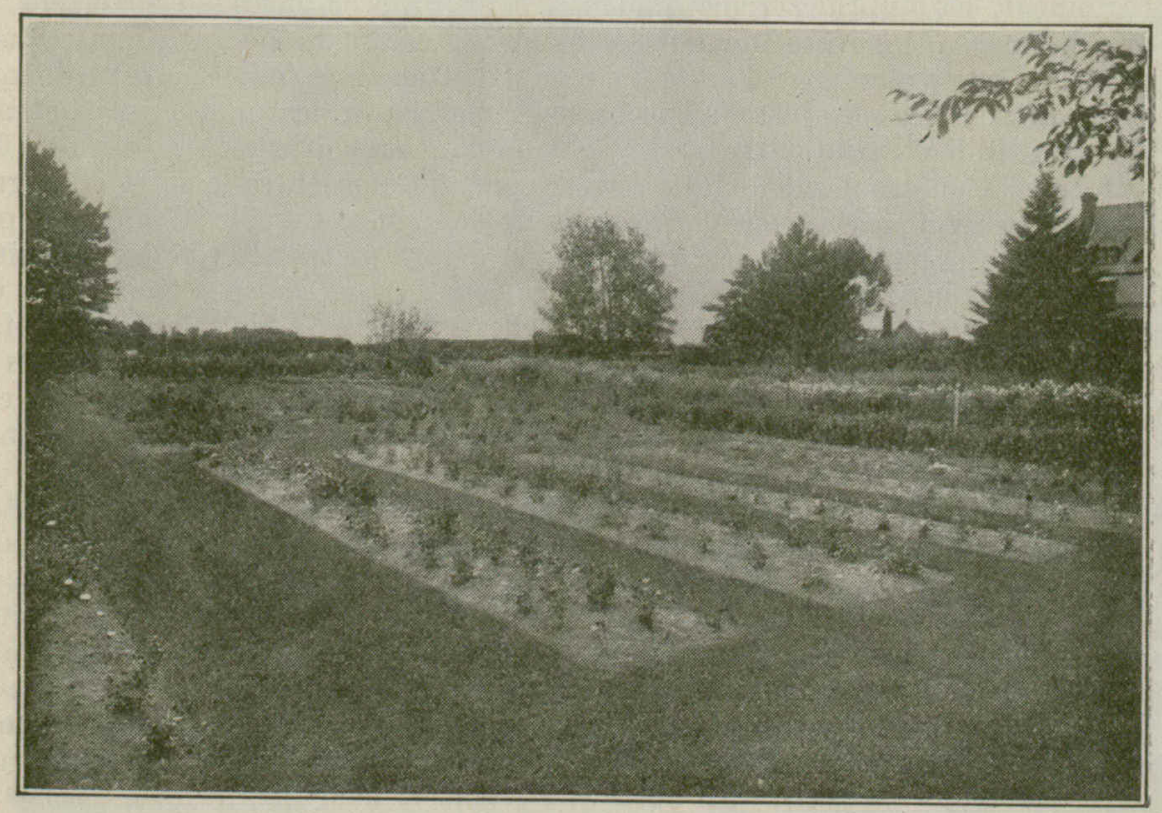

Moitié ouest de la roseraie, le premier été après la plantation.

des graviers ou de petites pierres à deux pieds de profondeur qui aideront à drainer le sol. Mettre au fond un bon sol de surface, pris ailleurs, de préférence un sol qui contient de l'argile, répandre là-dessus une forte couche de fumier pourri et l'incorporer à la bêche. On remet ensuite le sol de surface qui a été enlevé en premier lieu, ou du sol de meilleure qualité si celui-ci est pauvre, et on y incorpore de nouveau du fumier. On aura ainsi un bon terrain pour les roses. On laissera le sol se tasser pendant un mois avant de planter. C'est ce mode de préparation qui donne les meilleurs résultats, mais il n'est pas indispensable, et personne ne devrait s'abstenir de cultiver des roses sous prétexte qu'on ne peut prendre tous les soins. Les rosiers viennent bien dans presque tous les' sols gras, pourvu qu'ils ne soient pas très humides ni très secs.

\section{PLANTS ET PLANTATION}

Les plants vigoureux de deux ans et bourgeonnés sont les meilleurs. Les plants d'un an coûtent moins cher mais ils donnent peu de fleurs la première 
année et l'on est porté à se décourager avant le retour de la saison. Certains rosiers font très bien sur leurs propres racines, tandis que d'autres ne sont pas très vigoureux. Somme toute, les plants bourgeonnés sont les meilleurs. L'automne est la meilleure époque pour planter les rosiers, de préférence juste avant l'hiver. Parfois, cependant, les rosiers commandés pour l'automne ne sont pas livrés à temps; dans ce cas il est bon de les enfouir dans une terre non gelée, en enlevant à la bêche la terre gelée et en enterrant les racines et une forte proportion des tiges. Mais le novice dans cette culture est beaucoup plus sujet à se procurer ses roses au printemps, et c'est pourquoi tant d'échecs se produisent. Au moment où les plants arrivent à destination ils ont souvent commencé à se faner; plantés en cet état ils meurent presque infailliblement. Les conditions sont encore bien pires quand les plants arrivent en mai, par une température chaude, et quand les vents et la terre sont secs. Les roses thé doivent être plantées à environ quinze pouces d'écartement, les roses hybrides de thé à dix-huit pouces d'écartement, les hybrides remontantes à deux pieds et demi d'écartement, les hybrides Rugosa, les roses mousseuses et autres sortes rustiques à quatre pieds environ. Les roses plantées à l'automne ne doivent pas être taillées à cette époque; il vaut mieux les laisser jusqu'au printemps, mais si on les plante au printemps il faut les tailler vigoureusement à ce moment. Les arbrisseaux doivent être mis en terre à une profondeur telle que le point d'union entre le sujet et le greffon soit de deux à trois pouces au-dessous de la surface du sol. Ceci est important, car si le point d'union se trouvait près de la surface, le soleil durcirait le bois et gênerait la circulation de la sève. Ce sont là deux choses à éviter, car une végétation forte et vigoureuse est essentielle au succès. En plantant, il faut tasser le sol vigoureusement autour des plants afin de mettre les parcelles de terre en contact intime avec les racines pour que celles-ci puissent se procurer de l'eau aussi tôt que possible. Les rosiers ont relativement peu de racines et on ne doit en retrancher aucune avant la plantation, sauf les racines très longues qui pourraient gêner dans la plantation, ou encore les racines cassées. En plantant on évitera de laisser les racines exposées aux vents desséchants plus longtemps qu'il n'est absolument nécessaire. On trempe parfois les racines dans de la boue d'argile délayée dans de l'eau. Ce revêtement d'argile sur les racines non seulement protège ces dernières quand elles sont plantées, mais facilite, croyons-nous, la circulation de l'eau du sol aux racines. Si les tiges paraissaient fanées quand on les recoit, il faut enfouir les plants pendant deux jours dans une terre humide afin que les tiges puissent absorber de l'eau du sol. Cette précaution empêchera souvent les plants de périr. En outre il est bon, si la température est sèche au moment de la plantation, de ramasser la terre autour des tiges jusqu'à ce que la pluie tombe. Quand on plante en automne on doit ramasser la terre autour des tiges pour les protéger; on enlève cette terre au printemps et l'on étête fortement les plants. Il ne faut pas hésiter à tailler les tiges de façon à ne laisser que trois ou six bourgeons sur chaque tige qui sort de terre. Cette taille est encore plus rigoureuse dans le cas des plants établis au printemps; elle permet souvent de sauver des plants qui, sans ce retranchement, auraient sûrement péri.

\section{BINAGES ET ARROSAGES}

Nous avons déjà dit que les roses exigent beaucoup d'eau. En tenant la surface du sol bien meuble toute la saison, du printemps à l'automne, on retiendra dans le sol une quantité d'eau qui se serait évaporée si la surface du sol était restée ferme. Après chaque pluie il faut sarcler ou râtisser la surface, et dans une période de sécheresse, s'il est nécessaire d'arroser les roses, il faut ameublir le sol après avoir arrosé. Il vaut mieux arroser à fond, de temps à autre, que de donner des arrosages fréquents et légers. Il est bon cependant de seringuer ou de pulvériser les feuillages avec del'eau tous les jours, car c'est un des meilleurs moyens 


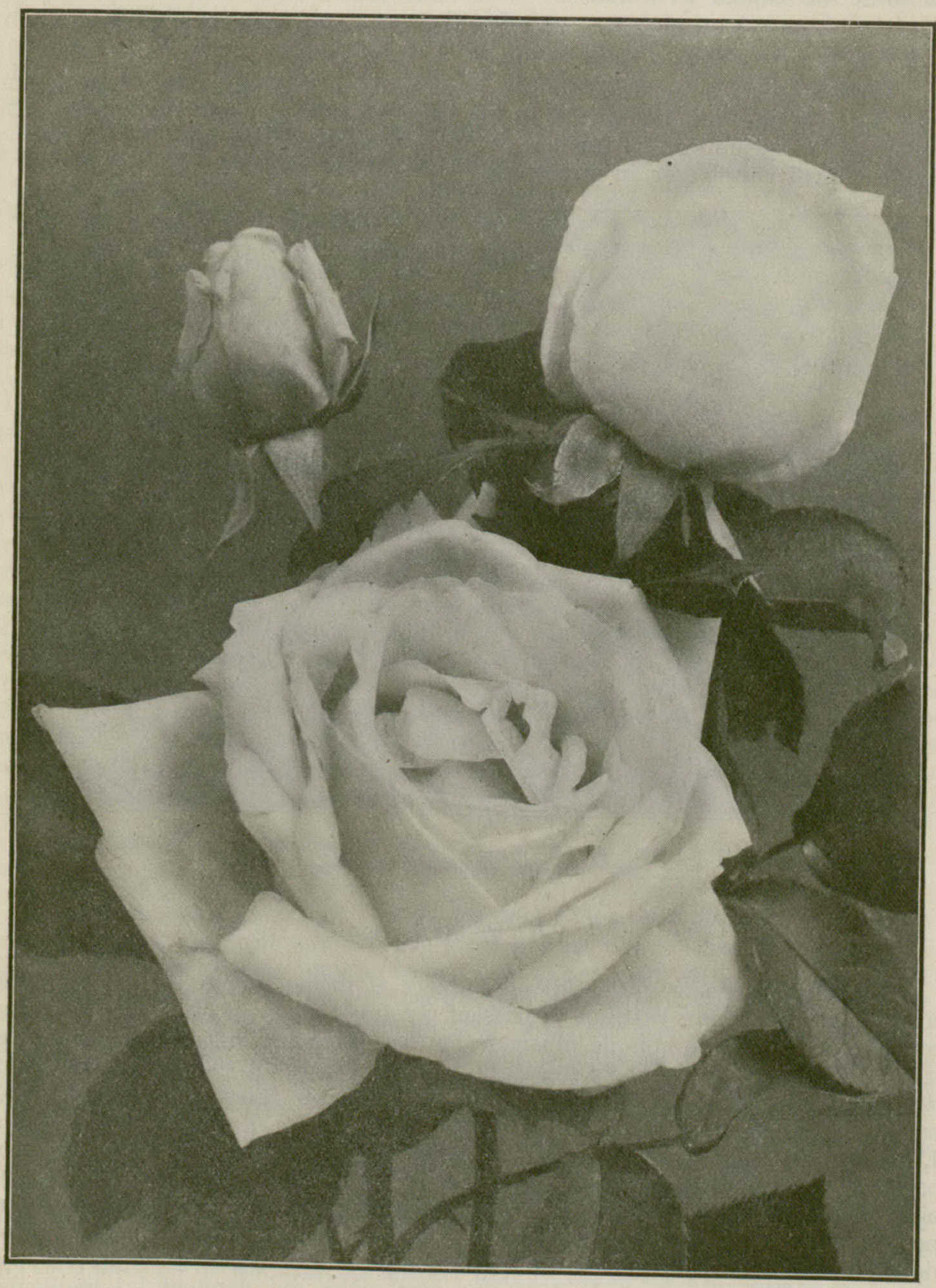

Frau Karl Druschk:-La rose blanche hybride remontante la meilleure et la plus sûre. 
de prévenir les insectes, mais cette pulvérisation a pour but de nettoyer le feuillage et non pas d'ajouter de l'humidité au sol. Elle doit être faite de bonne heure le matin, car le feuillage que l'on humecte au milieu d'une journée chaude et sèche est exposé à brûler.

\section{FUMURE}

On maintient la richesse du sol et sa provision d'humus en y appliquant tous les ans, une quantité généreuse de fumier de ferme bien décomposé. On peut épandre ce fumier à la surface du sol en automne et l'enfouir au printemps. On peut aussi l'appliquer au printemps.

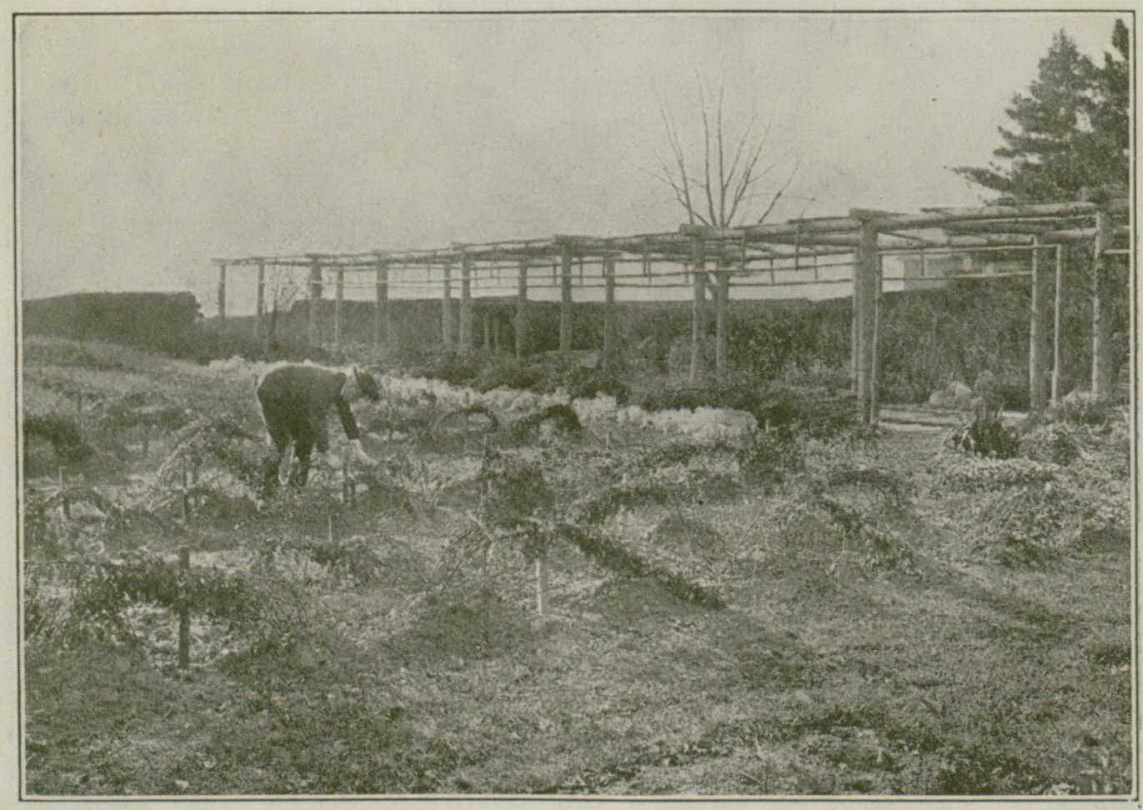

La préparation de la roseraie pour l'hiver. On attrache les plantes à des tuteurs juste avant de les recouvrir de branches d'épinettes.

\section{TAILLF}

La taille du rosier varie suivant l'espèce et même suivant la variété. Les roses du groupe le plus rustique fleurissent presque toutes sur le bois de la pousse précédente, ou sur du bois de plusieurs années, et comme on cultive principalement ces roses pour l'effet en masse de leurs fleurs plutôt que pour l'excellence de leurs fleurs prises séparément, il faut les tailler de façon à obtenir cet effet en masse. Ensuite, si on les taille presque jusqu'au sol, il y aura peu de roses. D'autre part, si on laisse les branches et les tiges de toute leur longueur, ou si on les étête seulement un peu de façon à avoir un buisson symétrique on aura un beau massif de roses. En outre, il est nécessaire de retrancher chaque année un peu du vieux bois. On coupe les branches au sol, on fait ainsi de la place et on donne accès à la lumière, ce qui permet aux jeunes tiges de se développer. Comme les tiges de un et de deux ans sont celles qui donnent les meilleures fleurs, il devrait y en avoir une forte proportion. Bien entendu, il faut enlever les branches mortes.

La plupart des roses du deuxième groupe doivent être taillées de façon différente, mais les roses grimpantes peuvent être traitées à peu près de la façon 
recommandée pour le premier groupe, à cette exception près que lorsque l'on désire revêtir un mur d'un grand buisson de roses, on laisse les vieilles tiges plus longtemps afin d'obtenir des branches latérales. Les roses hybrides remontantes donnent leur meilleure floraison lorsqu'elles sont fortement taillées. Pour obtenir les plus belles fleurs il faut, sans trève, raccourcir les arbrisseaux chaque printemps jusqu'à six ou douze pouces du sol. Si, toutefois, on désire obtenir un effet en masse, il faut laisser les arbrisseaux plus longs, et quand le sol est exceptionnellement bon, les rosiers qui n'ont pas été taillés fortement donnent un grand nombre de belles fleurs. On apprend bien vite par l'expérience quel est le meilleur mode de taille dans les conditions particulières; les variétés plus fortes n'exigent pas une taille aussi rigoureuse que les variétés plus faibles; parfois, quand on taille une variété très vigoureuse presque jusqu'à terre, on obtient très peu de fleurs car la végétation très forte qui vient ensuite n'en porte pas. Il vaut mieux tailler de bonne heure au printemps plutôt qu'à l'automne, car on ne sait jamais comment une plante se comportera en hiver. En taillant on laisse les tiges les plus fortes et les plus saines et l'on retranche les tiges plus faibles au ras de terre. On laisse de préférence un bourgeon extérieur plutôt qu'un bourgeon intérieur comme bourgeon terminal, afin que la tige sortant de ce bourgeon pousse vers l'extérieur du rosier.

Les roses hybrides de thé se taillent comme les hybrides remontants mais pas généralement autant. Pour les roses thé, dont les tiges meurent généralement au Canada quand elles résistent à l'hiver, il faut enlever tout le bois mort ou endommagé, et les branches non endommagées doivent être taillées sur un tiers de leur longueur. Il faut avoir grand soin d'enlever les rejetons ou les racines sortant du sujet sur lequel la rose est greffée. On les enlève aussitôt que l'on s'aperçoit de leur présence; il faut, pour cela, surveiller attentivement. On les coupe au ras du sujet et non pas au sol ou au-dessus du sol. Il peut être nécessaire, pour le faire, d'enlever un peu de terre. Il n'existe pas de moyen sûr de reconnaître le sujet mais on apprend par l'expérience; la vigilance est nécessaire, sinon le sujet reprend ses droits et bientôt la rose que l'on croit cultiver n'est plus là. Les feuilles du sujet sont souvent d'une couleur vert plus pâle que les variétés nommées et pas aussi luisantes. Parfois elles ont une teinte pourprée. Généralement les sujets ont un plus grand nombre de folioles sur les feuilles que les variétés nommées.

\section{PROTECTION EN HIVER}

Dans bien des parties du Canada les rosiers du premier groupe n'exigent que peu ou point de protection. Dans les provinces des prairies, où la terre est nue, il est bon d'en coucher quelques-uns et de les recouvrir de terre et également de branches d'arbres à feuilles persistantes si l'on peut en obtenir. En enlevant un peu de terre d'un côté du rosier, on peut le mettre à plat afin de pouvoir le recouvrir entièrement. Si la terre gelait et que la neige survienne avant que l'on ait recouvert l'arbrisseau, un bon moyen serait d'attacher les branches ensemble et de les recouvrir de branches à feuilles persistantes, de toile, ou de matériaux quelconques qui les protégeront contre le vent et le soleil. Ces matériaux doivent être de telle nature qu'ils puissent protéger les plantes sans retenir longtemps l'humidité, car les tiges de rosiers qui resteraient longtemps humides seraient exposées à moisir. Il est nécessaire de protéger les hybrides remontantes, les roses hybrides de thé et les roses thé. Un des moyens les plus simples de protéger les deux premières est de ramasser de la terre autour d'elles sur une hauteur de six à huit pouces ou plus en automne, protégeant ainsi la partie plus basse des tiges et si le dessus est détruit la partie basse reste presque toujours en vie. En outre, on peut coucher les plantes et les recouvrir de terre ou encore, quand les rosiers ne sont pas fortement taillés, on peut les recouvrir entièrement de terre pour les 
préserver. Si ce traitement ne suffisait pas, on pourrait mettre par-dessus la terre une couche légère de paille ou de branches à feuilles persistantes qui empêchera les gels et les dégels subits et parfois sauvera les plantes. Si le sol était gelé profondément avant que l'on pense à recouvrir les plantes, il faudrait coucher l'arbrisseau et le maintenir avec des planches que l'on recouvrirait de branches à feuilles persistantes. La neige même pourrait suffire à les protéger. $\mathrm{Au}$ printemps on aplanit le sol et l'on soulève les rosiers aussi tôt que possible pour éviter le développement de maladies. Si cette méthode ne réussissait pas. on pourrait coucher les rosiers et les recouvrir d'une caisse. On réussirait encore mieux en les couchant puis en les recouvrant d'une caisse sans fond et en remplissant cette caisse de feuilles sèches puis en la recouvrant d'un couvercle étanche. Mais si les feuilles sont humides quand les rosiers sont mis en caisse, ou si elles deviennent humides au printemps, les tiges seront exposées à moisir. Quand on se sert de caisse il faut enlever le couvercle à la première occasion au printemps pour permettre à l'air de circuler librement, afin que les tiges puissent sécher et l'écorce se durcir un peu avant que la caisse soit complètement enlevée.

Quant aux roses grimpantes on peut les descendre, lier les branches ensemble et les mettre dans une longue caisse étroite que l'on traite de la façon qui vient d'être décrite, si on constate que cette précaution est nécessaire. On peut aussi, dans les localités où il y a beaucoup de neige, les coucher et les recouvrir complètement ou partiellement de terre ou de branches d'épinette, comme pour les roses naines. Les roses thé sont les plus délicates; on doit les recouvrir de terre comme les roses hybrides perpétuelles et les roses hybrides de thé; on obtiendra les meilleurs résultats en les recouvrant d'une caisse remplie de feuilles sèches comme nous venons de dire. La méthode à adopter dépendra de la partie du Canada où l'on demeure, mais une des méthodes décrites devrait suffire dans la plupart des conditions.

\section{INSECTES ET MALADIES FONG EUSES - MOYEN DE LES COMBATTRE}

Il ne saurait y avoir de belles roses sans bon feuillage; en outre, le bon feuillage fait bon effet dans un jardin de roses. Les feuilles fortement recroquevillées, recouvertes de blanc, tachetées ou mangées, détériorent beaucoup l'aspect du jardin. L'insecte le plus facile à combattre est la limace des roses, une chenille verte qui ne se montre pas généralement en grand nombre, mais qui opère à la surface inférieure des feuilles et en enlève des morceaux. On peut les enlever à la main quand les rosiers sont peu nombreux, mais le vert de Paris ou l'ellébore pulvérisé sur le rosier, de façon à atteindre la partie inférieure des feuilles, les tue promptement. Le vert de Paris employé devrait être faible de façon à ne pas brûler le feuillage, une bonne proportion est d'une once par 12 gallons d'eau. On emploie l'ellébore dans la proportion d'une once par deux gallons d'eau.

Le puceron ou la mouche verte cause parfois de grands ennuis et le thrips, un petit insecte sauteur qui fait recroqueviller les feuilles, fait aussi beaucoup de mal si on ne prend les moyens de l'enrayer. Il existe de bons remèdes pour ces insectes, et comme il vaut mieux prévenir que guérir, il faut appliquer ces remèdes avant que ces insectes ne se soient propagés. Nous avons déjà dit qu'un lavage à l'eau seule est un bon moyen de prévention. On peut employer de la nicotine dans la proportion d'une cuillerée à thé dans un gallon d'eau ou une décoction faite de morceaux de quassia et de savon mou, ou de savon d'huile de baleine que l'on obtient en faisant bouillir quatre onces de morceaux de quassia pendant dix minutes dans un gallon d'eau douce, puis on filtre tandis que le liquide est encore chaud et l'on y fait dissoudre quatre onces de savon d'huile de baleine, puis on ajoute un gallon d'eau. Il peut être bon de seringuer avec de l'eau après que les insectes sont tués pour nettoyer les plantes.

Le savon d'huile de baleine, dans la proportion de une livre dans six gallons d'eau, est un bon insecticide à employer contre les pucerons et les thrips. 


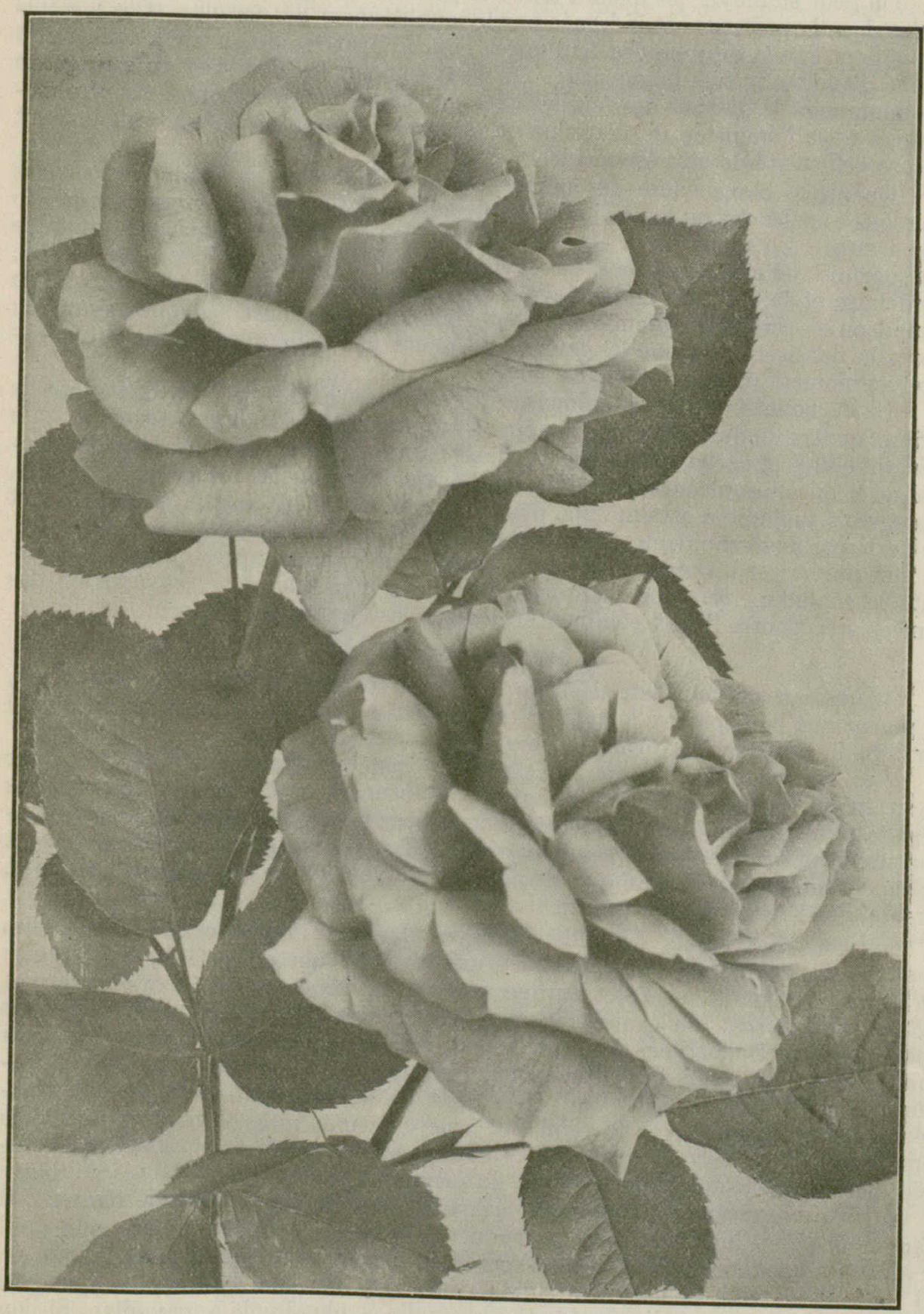

Hugh Dickson-Une des meilleures roses hybrides remontantes. 
L'émulsion de kérosène (huile de charbon) est un remède sûr, mais elle peut abîmer le feuillage si elle n'est pas bien faite.

On peut employer les mêmes remèdes pour les araignées rouges, de petits insectes dont la présence est indiquée par le jaunissement des feuilles. Ces insectes travaillent sur le dessous des feuilles et l'on ne peut les découvrir à l'œil nu, à moins d'avoir une très bonne vue. On peut souvent les maîtriser en seringuant abondamment le dessous des feuilles avee de l'eau seule; des fleurs de soufre mélangées avec l'émulsion de kérosène ou de savon d'huile de baleine augmentent encore l'efficacité de ces insecticides.

Quand le blanc cause des ennuis on peut l'enrayer en répandant sur les buissons tous les dix ou douze jours des fleurs de soufre jusqu'à ce que la maladie disparaisse. La tache noire (Leaf blotch, ou black spot) est une autre maladie qui parfois défigure beaucoup les feuilles et affaiblit les plantes. La boúillie bordelaise et la solution ammoniacale de carbonate de cuivre l'arrêteront, mais quand on se sert de la première il faut l'employer plusieurs semaines avant ou après la floraison, afin que les feuilles ne soient pas défigurées par la pulvérisation au moment où ce rosier est en fleurs.

Le carbonate de cuivre ammoniacal se fait avec une once de carbonate de cuivre, quatre cinquièmes d'une chopine d'ammoniaque et huit gallons d'eau. On fait dissoudre le carbonate de cuivre dans l'ammoniaque. On conserve l'ammoniaque et la solution concentrée dans des bocaux bien bouchés, de verre ou de grès. Lorsque la maladie a attaqué une plantation, on doit s'efforcer de la maitriser et de l'extirper aussi rapidement que possible au moyen de pulvérisations bien faites. taté la maladie. Sur une plantation où elle a déjà exercé ses ravages en des années précédentes il faut commencer dès que les feuilles se montrent.

\section{MULTIPLICATION}

L'amateur enthousiaste désirera sûrement multiplier ses rosiers lui-même; le plaisir que l'on éprouve à cultiver cette superbe fleur est encore plus vif quand on peut la propager soi-même.

Les rosiers qui poussent en plein air sont généralement multipliés par la greffe en écusson ou l'écussonnage. Le bouturage ne donne pas de très bons résultats au Canada; on peut cependant se servir de cette méthode assez avantageusement connue sur certaines variétés. Les autres genres de greffage sont rarement employés en plein air; ils ne se pratiquent guère que dans les serres et encore d'une façon très restreinte.

Sujets.-Les sujets les plus employés dans la multiplication des rosiers sont le rosier églantier (Rosa canina), une rose sauvage de la Grande-Bretagne, et le rosier Manetti, un sujet européen d'origine italienne. L'églantier odorant (Rosa rubiginosa), fait également un très bon sujet. Il est possible que certains rosiers sauvages canadiens, à pousse vigoureuse, feraient de très bons sujets. On multiplie les églantiers par l'écussonnage ou par voie de semis. On peut se procurer, à très bas prix, chez les pépiniéristes, cảa boutures enracinées. L'amateur peut lui-même les faire venir par voie de semis, mais il gagnera au moins une année en se servant de boutures enracinées. Les sujjets enracinés qu'il reçoit généralement au commencement du printemps doivent être plantés au moins à un pied d'espacement, en rangées écartées de trois pieds l'une de l'autre. Il faut tenir le sol bien biné pour provoquer la pousse rapide des plants, afin qu'il y ait une abondance de sève sous l'écorce au moment où l'on fera l'écussonnage. Il faut aussi tenir la base des sujets rechaussée sur une hauteur de quelques pouces en été, pour que l'écorce reste plus molle et plus facile à travailler. Si l'on cultive des sujets par voie de semis, il faut semer en automne, en rangées espacées d'environ six pouces dans un sol bien préparé. Les jeunes plantes paraîtront au commencement du printemps. Elles seront devenues, en automne, de jolies petites plantes, si T'on a eu le soin de tenir la surface du sol bien meuble. 
Avant l'hiver ou au commencement du printemps, il faut les transplanter en rangs de pépinière, comme nous venons de décrire pour les sujets importés. Quelques-unes d'entre elles devraient alors être prêtes pour l'écussonnage cette saison. On ne conserve qu'une tige principale, mais avec tous les rameaux latéraux. Généralement les amateurs réussissent mieux l'écussonnage en se servant du sujet Manetti mais ce sujet n'est pas aussi avantageux que l'églantier, car

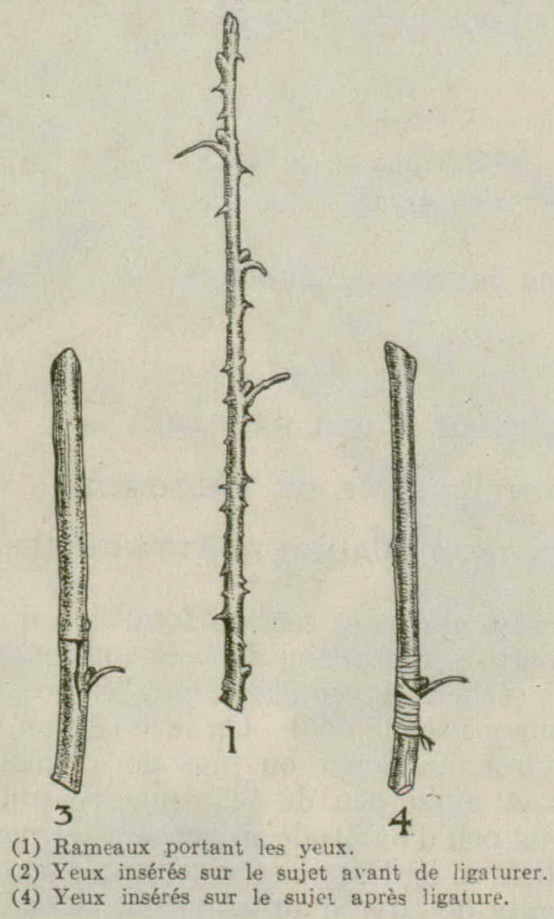

le feuillage des sujets ne se distingue pas facilement de celui de quelques variétés: nommées de roses. En outre, les roses ne durent pas aussi longtemps sur le Manetti que sur l'églantier. Lorsqu'on veut greffer des rosiers de variétés régulières, qui ne peuvent être cultivés que dans les parties les moins rigoureuses du Canada, il faut attendre, pour faire l'écussonnage, que les sujets aient au moins. un an de plus que lorsque l'on greffe des rosiers nains ordinaires.

Ecussonnage.-L'écussonnage des rosiers est très semblable à l'écussonnage des arbres fruitiers. Tous ceux qui sont adroits de leurs mains peuvent très bien faire cette opération. L'époque de l'écussonnage varie quelque peu suivant la localité; les sujets sont à point, suivant les régions du Canada, à partir de law fin de juillet jusqu'au commencement de septembre. On commence à écussonner dès que l'on peut se procurer de bons yeux. Les sujets sont prêts à tout moment à partir de cette époque; il ne faut pas attendre que la pousse se soit ralentie à un tel point qu'il ne reste plus assez de sève pour permettre de soulever facilement l'écorce. Lorsque la pousse est assez rapide, on n'a aucune difficulté. Les yeux sont en bon état lorsqu'ils sont bien développés, mais encore dormants et que le bois sur lequel ils se trouvent est ferme, et dans l'état que l'on peut appeler semi-aoûté. Au commencement de la saison le sujet est à point pour le greffage lorsqu'une tige a fleuri.' Plus tard dans la saison, les yeux seront suffisamment développés sur les tiges dont les fleurs ne se sont pas encore ouvertes. A l'époque convenable, on coupe des morceaux de tiges portant plusieurs bons yeux et immédiatement après les avoir coupés, on enlève toutes les feuilles pour empếcher l'évaporation d'humidité, mais en laissant environ un pouce du pétiole de la feuille attaché à la tige. On tient ces rameaux enveloppés dans de la mousse ou. 
dans des sacs humides pour les empêcher de sécher, car il faut que les yeux soient tenus frais. On prépare ensuite les sujets en enlevant le sol autour d'eux jusqu'à une profondeur de deux pouces au-dessous de la surface ou aussi près des racines que possible; on nettoie la tige et on enlève tous les rameaux qui pourraient gêner. On fait alors une incision en travers de l'écorce en pénétrant jusqu'au bois, et une autre incision longitudinale d'environ un pouce de long, partant de la première. Les deux incisions ont quelque peu la forme $\mathrm{T}$.
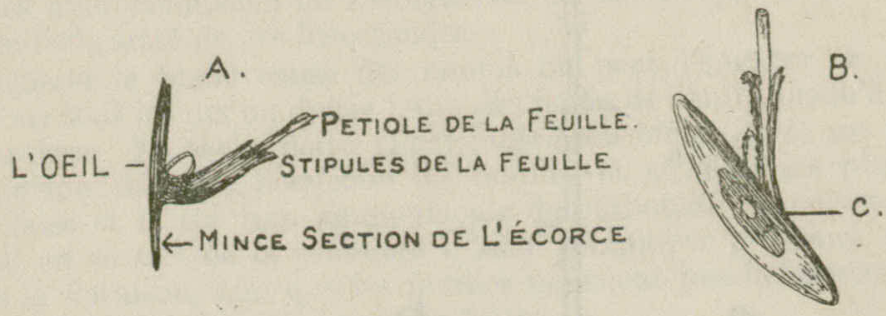

\section{A. VUE de Côté de L'oeil préparé. \\ B. Surface intérieure de L'écorce. C. Mince Section d'aubier attachée à L'ecorce.}

Comme l'été peut être chaud et sec, on fera bien d'insérer les yeux sur le côté nord-est du sujet, où ils seront moins exposés aux rayons du soleil et par conséquent moins exposés à sécher. On soulève alors l'écorce au moyen de la spatule en ivoire du greffoir pour y insérer l'œil. On lève cet œil sur le rameau avec un lambeau d'écorce d'environ un pouce ou plus de longueur-un demi-pouce de chaque côté de l'œil,- et aussi peu de bois que possible. Ce bois doit être enlevé. Ceci demande un peu d'habitude si l'on ne veut pas endommager l'œil ou l'écorce. On enlève le bois en le détachant vivement et en s'aidant de la lame du couteau. Il est nécessaire de tirer d'un coup sec, sinon l'œil pourrait être entraîné avec le bois et abîmé. Si le bois ne se détache pas facilement c'est que l'œil est trop mûr; dans ce cas, il faudra se procurer du bois plus jeune. Toutefois si l'écusşon que l'on a levé ne contient qu'une infime quantité de bois' il n'y aura pas d'inconvénient à le laisser. L'opération réussira aussi bien. Après avoir enlevé le morceau de bois, on taille l'écorce aux deux bouts pour lui donner la longueur voulue et on l'insère sous l'écorce du sujet en se servant du pétiole de la feuille en guise de poignée, et en soulevant l'écorce de l'autre main avec le 'couteau. Il ne faut pas laisser l'œil exposé à l'air sec plus longtemps qu'il n'est absolument nécessaire car il ne faut pas qu'il sèche. L'œil, une fois mis en place, doit se trouver juste à l'endroit où les deux incisions se croisent. On ligature alors avec du raphia que l'on serre étroitement autour de la tige, au-dessus et au-dessous de l'œil. On apprendra bien vite à faire cette opération convenablement. Au bout de trois ou quatre semaines, si l'opération a été bien faite, l'œil aura repris, et si l'on a tenu la terre humide, le raphia aura pourri et n'aura pas besoin d'être détaché. Si, cependant, le raphia est encore serré, il faudra le couper au bout d'un mois car il gênerait la circulation de la sève. On laisse le sujet pousser comme d'habitude jusqu'à ce que la végétation s'arrête. Au commencement du printemps, on écime le sujet jusqu'à trois ou quatre pouces de l'œil, sur les plantes où l'opération a réussi. Dès que les yeux se mettent à pousser, il faut bien les surveiller car les nouveaux rameaux sont exposés à se rompre, à moins qu'ils ne soient attachés. Il est bon d'avoir de forts bâtons ou des cannes de bambou pour y fixer les rameaux, à mesure qu'ils poussent. On écime, au fur et à mesure, toute la pousse des sujets, et l'on enlève les moignons des 


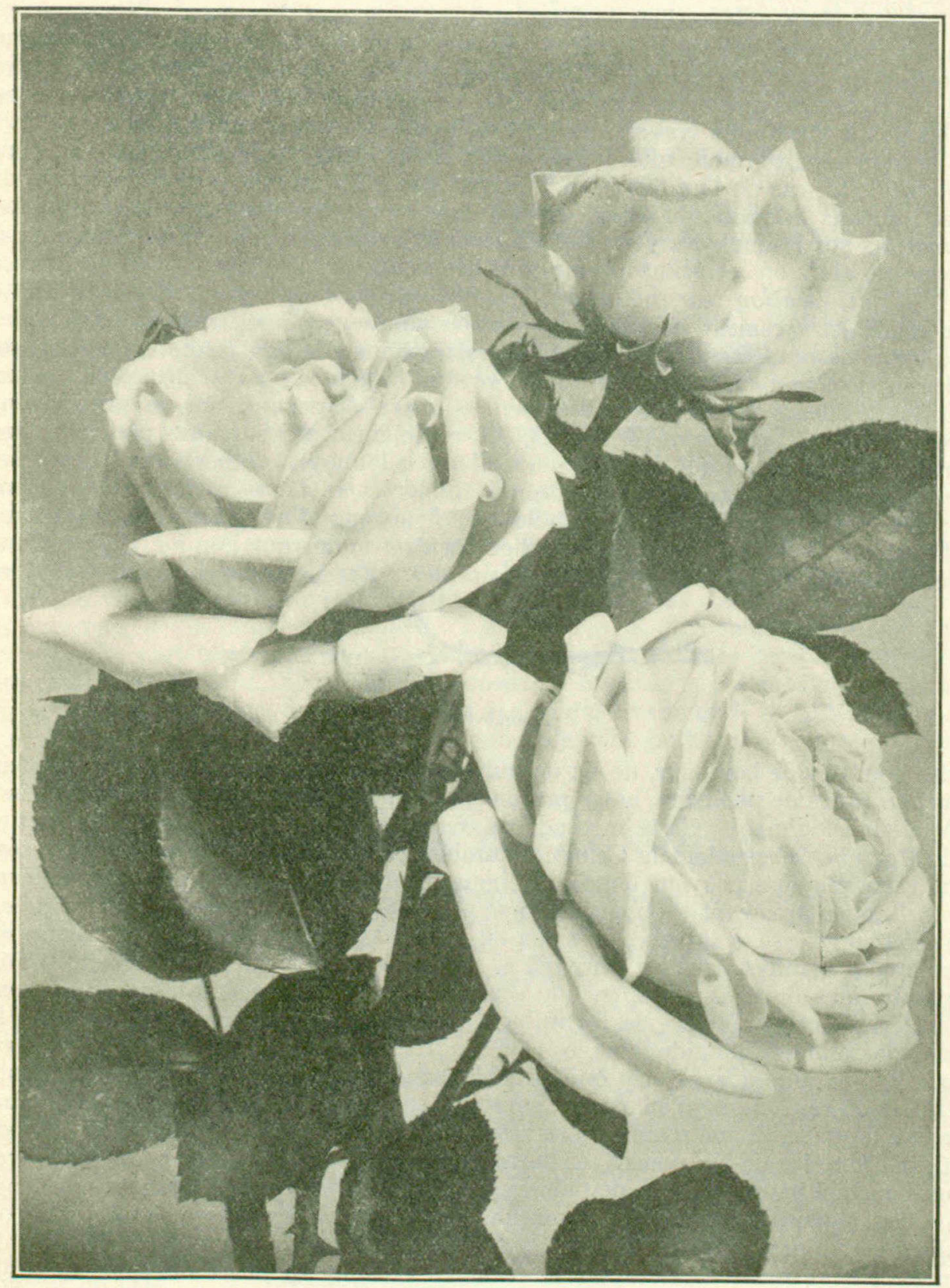

Caroline Testout-Rose hybride de thé, très sûre. 
sujets dès que l'on peut attacher les nouveaux rameaux. Ces sujets sont prêts à être transplantées dans la roseraie après une saison de végétation. Pour les rosiers des variétés régulières, l'écussonnage se fait à peu près de la même façon que pour les rosiers nains sauf ce point que les yeux sont insérés près du sommet de la tige principale au lieu de l'être au pied. Lorsque l'époque de l'écussonnage arrive, on insère les yeux sur deux branches latérales de la pousse de la saison, sortant de la tige principale à la hauteur désirée. On insère les yeux très près de la tige principale. Généralement, en écussonnant les rosiers réguliers, on ne fait pas d'entaille en travers, de peur de casser les petits rameaux mais on fait l'incision longitudinale plus longue. On insère deux yeux dans deux rameaux latéraux, au cas où l'un d'eux ne viendrait pas. En automne, on retranche tous les rameaux latéraux, on écime jusqu'à trois ou quatre pouces du sujet les deux rameaux sur lesquels les yeux ont été insérés jusqu'à ce qu'ils aient commencé à pousser, alors on les écime jusque près des yeux.

Multiplication par bouturage (Roses sur leurs propres racines).-Cette méthode est vivement recommandée par les amateurs insouciants qui laissent les sujets pousser et étouffer la variété écussonnée mais le bon jardinier se contente des sujets écussonnés, car il sait qu'en général la pousse sera beaucoup plus forte, et que les fleurs seront plus belles, sur des rosiers écussonnés sur un sujet vigoureux comme l'églantier. Toutefois, les renseignements suivants pourront être utiles aux amateurs qui désirent multiplier par le moyen de boutures. Certaines roses grimpantes comme la Crimson Rambler et la Dorothy Perkins viennent facilement par le bouturage, tandis que beaucoup d'hybrides remontantes et d'hybrides de thé viennent assez difficilement, à moins que l'on ne dispose d'une serre offrant toutes les conditions désirables. La méthode consiste à prendre

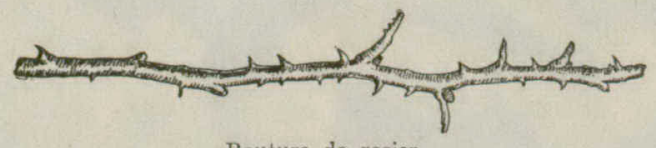

Bouture de rosier.

des boutures de bois mûr, de six ou huit pouces de long, et à les planter en plein air, en rangs de pépinière, assez profondément pour qu'un seul bourgeon paraisse à la surface du sol. Cette méthode ne réussit guère dans la plupart des parties du Canada. Cependant la Crimson Rambler et certaines autres variétés traitées de cette manière peuvent donner de bons résultats. On obtiendra les meilleurs résultats en prélevant les boutures lorsque le bois est à peu près à moitié aoûté en juillet; on laisse, à la base, un talon ou un petit morceau de bois plus vieux; on enlève les feuilles, sauf les deux qui se trouvent les plus près du sommet, et l'on plante dans du gros sable, en une couche froide. Mais même lorsqu'elles sont traitées de cette manière, les boutures s'enracinent difficilement. Il faut tenir le sol constamment humide dans les couches froides, et tenir l'air aussi humide que possible en aérant aussi peu que possible, sans toutefois provoquer la moisissure des boutures. Lorsqu'on désire enraciner un petit nombre de boutures, une méthode qui a donné d'assez bons résultats est la suivante: on prend un pot de fleur ordinaire de six pouces, en terre poreuse; on bouche le trou au fond avec un bouchon pour que l'eau ne puisse en sortir. On met ce pot dans un autre pot de neuf pouces et on remplit l'espace entre les deux pots avec du gros sable: on dispose d'abord au fond quelques gros matériaux, par exemple des morceaux de pots de fleur ou des scories, pour le drainage, puis une quantité suffisante de sable pour que le petit pot se trouve à bonne hauteur. Le dessus de ce petit pot devrait être à environ un pouce du dessus du gros pot. On plante ensuite les boutures à un pouce ou deux d'écartement dans le sable, on remplit le pot de six pouces avec de l'eau et on le met dans un endroit où il sera exposé aux rayons du soleil pendant le plus grand nombre d'heures possible, par jour. Le sol sera maintenu 
suffisamment humide si l'on tient toujours le petit pot rempli d'eau et les conditions seront très favorables à l'enracinement des boutures. Si on laissait le sable sécher, naturellement les boutures seraient perdues.

Marcottage.-Certaines variétés de roses se multiplient facilement au moyen du marcottage. Le marcottage consiste à coucher les rameaux et à recouvrir la partie qui est couchée avec une couche de terre de quatre à six pouces de profondeur. On laisse le bout et la partie supérieure des branches exposés. Avec le temps, la branche s'enracine à l'endroit où elle est courbée, et l'on sectionne alors la partie enracinée. Généralement la branche s'enracine beaucoup plus vite; on la sectionne en partie, à travers la base d'un bourgeon, à l'endroit où elle est courbée, et si l'on fait une autre entaille d'un pouce ou plus, dans le sens de la longueur de la branche, à travers le bourgeon. Les branches ainsi traitées s'enracinent parfois très vite, surtout si l'on tient cette entaille couverte en y mettant un peu de gros sable.

Rejets.-Certaines roses qui poussent souvent par leurs propres racines comme la rose jaune de Perse, la rose japonaise (Rosa rugosa), la rose de Provence et la rose de Damas se multiplient rapidement au moyen des rejets qui poussent autour de la plante mère et que l'on arrache pour les replanter.

Création de nouvelles variétés.-La création de nouvelles variétés de roses est une étude très intéressante; tous les amateurs peuvent espérer obtenir de meilleures variétés que celles qui existent déjà, mais il est douteux cependant que ce soit faire un bon emploi de sa terre lorsqu'on ne dispose que d'une petite étendue de terrain, car il n'y a qu'une faible proportion de plantes de semis qui produisent de bonnes roses et ce n'est qu'au bout de trois ou quatre ans que l'on peut connaître le résultat de son travail. Le moyen le plus simple de créer de nouvelles variétés est de conserver la graine des meilleures sortes et de la semer en automne, de la manière indiquée. La méthode la plus intéressante est de croiser deux variétés ayant les caractéristiques que l'on désire combiner en une seule. En peu de mots, cette méthode consiste à déposer le pollen d'une variété sur le pistil d'une autre, dont le pollen a été enlevé avant qu'il se soit répandu. Les détails de l'opération sont décrits dans d'autres bulletins de la série des fermes expérimentales.

\section{BONNES ROSES DE JARDIN Ā OTTAWA}

La plupart des roses dont les noms sont donnés dans les listes suivantes se trouvent dans la nouvelle roseraie de la ferme expérimentale centrale, à Ottawa.

Ces listes ont été revisées et agrandies dans cette édition.

Toutes les roses recommandées qui paraissent sur ces listes ont donné la preuve de leurs mérites dans des essais faits à Ottawa au cours de ces dernières années. Beaucoup de ces roses sont hautement recommandées par les amateurs en Grande-Bretagne, sur le continent européen et sur ce continent.

Parce qu'une rose est en grande valeur et qu'elle est recommandée en Europe et aux Etats-Unis, ceci ne veut pas toujours dire que ce soit celle qui convienne le mieux au Canada. Nous sommes toujours heureux, lorsque nous constatons, au cours de nos essais à Ottawa, qu'une rose maintient chez nous la bonne réputation dont elle jouit ailleurs. Souvent, toutefois, une rose bien réputée ailleurs ne réussit pas à Ottawa, peut être parce que l'une ou l'autre de ses qualités essentielles ou héréditaires ne résiste pas au froid de l'hiver ou à la chaleur de l'été.

Il se met sur le marché tous les ans un grand nombre de nouvelles roses hybrides et nous en essayons le plus possible à Ottawa. Nous reviserons les listes suivantes à mesures que nous serons mieux renseignés sur l'adaptation relative des variétés à certaines conditions. 
BONNES ROSES HYBRIDES DE THÉ POUR LE JARDIN

\begin{tabular}{|c|c|c|}
\hline Nom & $\begin{array}{c}\text { Date } \\
\text { d'origine }\end{array}$ & Description \\
\hline 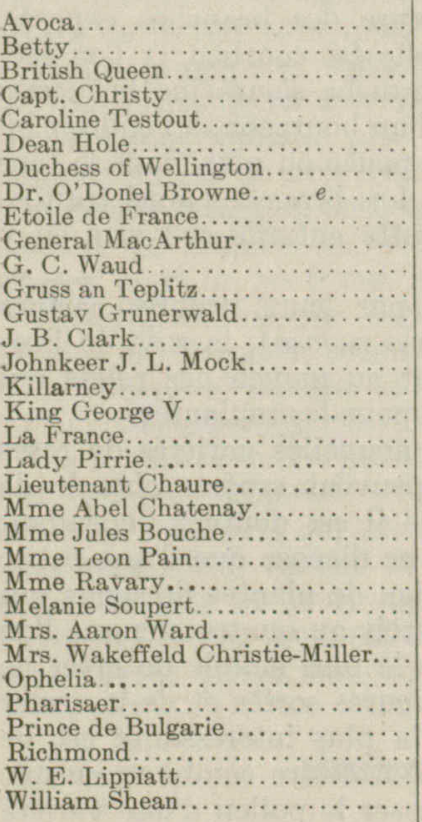 & $\begin{array}{l}1907 \\
1905 \\
1912 \\
1873 \\
1890 \\
1904 \\
1909 \\
1908 \\
1904 \\
1905 \\
1908 \\
1897 \\
1903 \\
1905 \\
1909 \\
1898 \\
1912 \\
1867 \\
1910 \\
1910 \\
1895 \\
1911 \\
1904 \\
1899 \\
1905 \\
1907 \\
1909 \\
1912 \\
1901 \\
1902 \\
1905 \\
1907 \\
1906\end{array}$ & $\begin{array}{l}\text { Cramoisi riche, odorante. } \\
\text { Rose de cuivre, ombragée de jaune, odorante. } \\
\text { Blane crémeux. } \\
\text { Couleur de chaire délicate, plus prononcée au centre. } \\
\text { Rose brillant. } \\
\text { Rose pâle argenté. } \\
\text { Jaune safran foncé, pétales extérieures orange. } \\
\text { Rose carmin, odorante. } \\
\text { Cramoisi velouté, odorante. } \\
\text { Cramoisi écarlate, brillant, odorante. } \\
\text { Rose, éclaboussé d'orange et écarlate. } \\
\text { Cramoisi brillant, odorante. } \\
\text { Rose carmin, jaune à la base. } \\
\text { Cramoisi écarlate, ombragée de noir. } \\
\text { Rose foncé, pétales extérieures carmin. } \\
\text { Couleur de chair, éclaboussée de rose pâle, odorante. } \\
\text { Cramoisi foncé. } \\
\text { Rose argenté, odorante. } \\
\text { Rose de cuivre, délicat. } \\
\text { Cramoisi riche, odorante. } \\
\text { Rose saumon. } \\
\text { Blane. } \\
\text { Couleur de chair argenté. } \\
\text { Jaune orange pâle. } \\
\text { Jaune pâle (coueher du soleil). } \\
\text { Jaune indien, bords des pétales blancs. } \\
\text { Rouge tendre, pétales rose foncé à l'extérieur. } \\
\text { Couleur de chair saumon, odorante. } \\
\text { Rose blanchâtre, ombragé de saumon pâle. } \\
\text { Rose pâle teinté, ombragé abricot. } \\
\text { Cramoisi pâle brillant. } \\
\text { Vramoisi velouté. } \\
\text { Rose crémeux. }\end{array}$ \\
\hline
\end{tabular}

\section{BONNES NOUVELLES ROSES THÉ HYBRIDES}

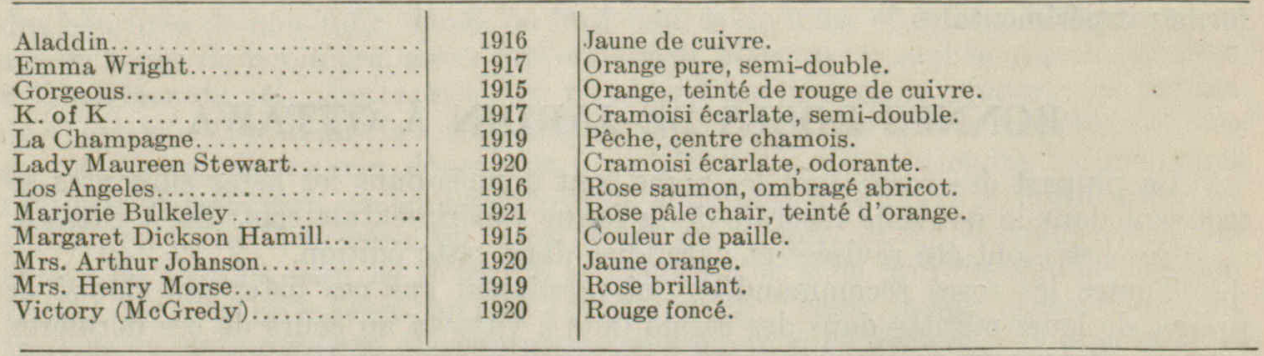

\section{BONNES ROSES HYBRIDES VIVACES}

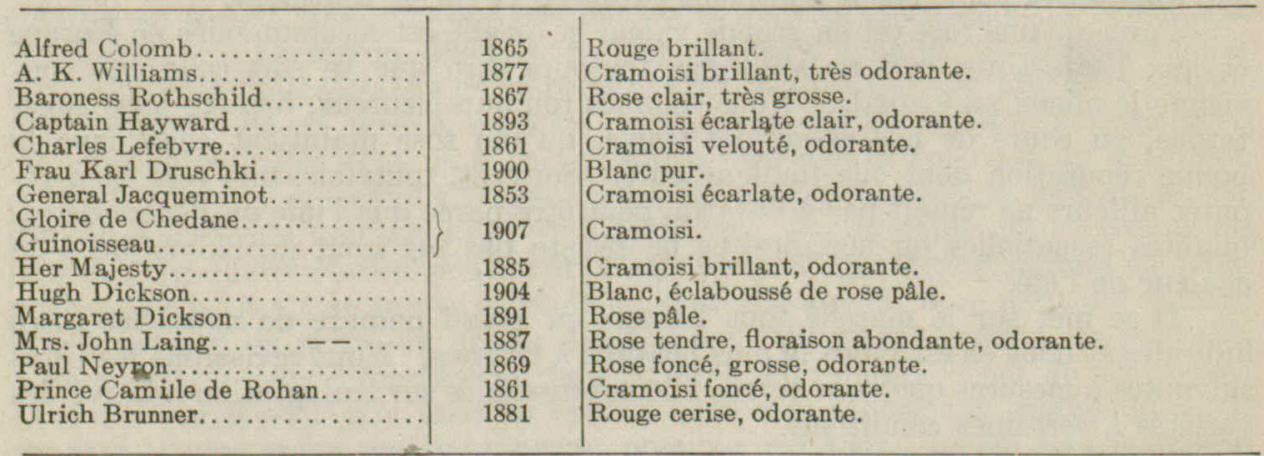




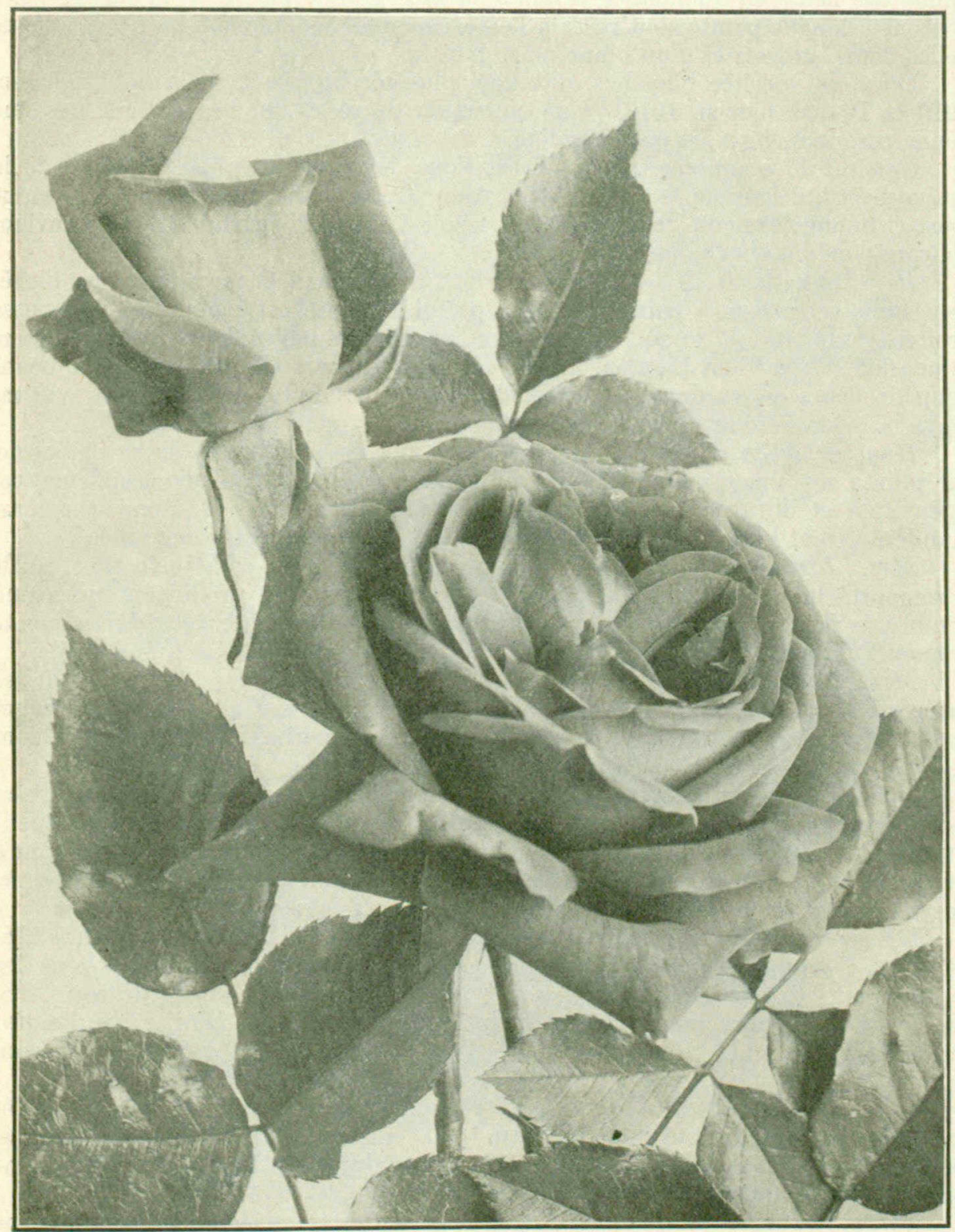

General MacArthur-Une très bonne rose hybride de thé, rouge cramoisi. 


\section{Collection spéciale de roses rustiques pour le petit jardin}

Frau Karl Druschki, H. R.-Il est admis que cette rose est la plus belle rose blanche qui soit en culture. Le rosier a une pousse vigoureuse et saine, et donne des fleurs en abondance pendant la plus grande partie de la saison. Les boutons à longue pointe sont roses à l'extérieur mais ils dornent, en s'ouvrant, de belles fleurs, grosses et d'un blanc neige pur.

D'autres variétés blanches, d'origine plus récente, sont les Ethel Malcolm, 1910 et British Queen, 1912. Une collection de roses qui ne contient pas une bonne rose blanche n'est pas complète.

Général Jacqueminot, $H$. R.-C'est l'une des roses les plus anciennes de la catégorie et qui soutient encore sa réputation. Cette plante fait une pousse vigoureuse et donne des fleurs en abondance. Elle est cramoisi écarlat vif, mais devient pourpre pendant les chaleurs. Odorante.

Her Majesty, H. R.-Cette rose vient très bien à Ottawa, et nous l'insérons dans cette liste à cause de son type. La plante est vigoureuse, à pousse droite, et elle produit, lorsqu'elle s'est bien établie, de belles fleurs roses de bonne dimension. Elle vient bien en une saison chaude, mais elle ne donne pas beaucoup de fleurs en automne. Lorsqu'elle a été introduite, en 1885, elle a remporté la médaille d'or N. R. S. par acclamation.

Hugh Dickson, H. R.-Cette rose est l'une des meilleures de sa catégorie. La plante est vigoureuse et fleurit abondamment. La couleur des fleurs est. attrayante et durable; elle est cramoisi ombré d'écarlate. Hautement recommandée. Vient bien à Ottawa et généralement dans les jardins des villes.

Mrs. John Laing, H. R.-Une autre rose recherchée et à juste titre. Elle a remporté la médaille d'or. Rosier à pousse vigoureuse produisant un grand nombre de fleurs au printemps et également en automne. Fleurs rosâtres, bonne grosseur, de qualité durable. Odorante. Vient bien dans les jardins de ville.

Mrs. R. G. Sharman-Crawford, H. R.-C'est l'une des meilleures de sa catégorie pour une deuxième récolte de fleurs. Le rosier a un développement passable et il est sain. Les fleurs ont une teinte superbe, rosâtre clair, et sont de grosseur modérée. Cette variété est de culture facile.

Ulrich Brunner, H. R.-Cette rose appartient au groupe des trois roses recommandées par l'un des amateurs les plus renommés comme les "trois meilleures roses pour les jardins de cottage". Le rosier est vigoureux et vient bien à Ottawa. Il ne fleurit pas beaucoup mais produit quelques fleurs en automne. Les fleurs sont rouge-cerise, odorantes et de bonne grosseur.

Les sept variétés précédentes de roses hybrides sont toutes de couleur différente. Ce sont peut-être les meilleures de leur catégorie. Nous pouvons les recommander à tous ceux qui désirent cultiver un nombre limité de roses. Si l'on désire un nombre encore plus restreint on pourra faire un choix des suivants: Frau Karl Druschki (blanche), Mrs. John Laing (rose), et Hugh Dickson (rouge).

Caroline Testout, H. T.-Cette rose a été en grande faveur dans sa catégorie depuis qu'elle a été introduite en, 1890. Le rosier fait une bonne pousse. Les fleurs sont d'une couleur rose éclatant, assez durable. La floraison est abondante et sûre. Bonne pour les jardins de ville.

General MacArthur, H. T.-Une des meilleures roses thé hybrides d'origine américaine pour le jardin. Elle a une pousse vigoureuse et porte beaucoup de fleurs d'un rouge cramoisi vif et très odorante. Le feuillage est meilleur également que dans beaucoup de variétés. Sa fleur n'est pas aussi pleine que certaines autres sortes, mais elle a d'excellentes qualités qui rachètent ce défaut.

Gruss an Teplitz, H. T.-C'est là l'une des roses thé hybrides des plus rustiques et des plus vigoureuses et bien que les fleurs ne soient que semi-doubles et qu'elles ne soient pas aussi attrayantes comme fleurs coupées que beaucoup d'au- 


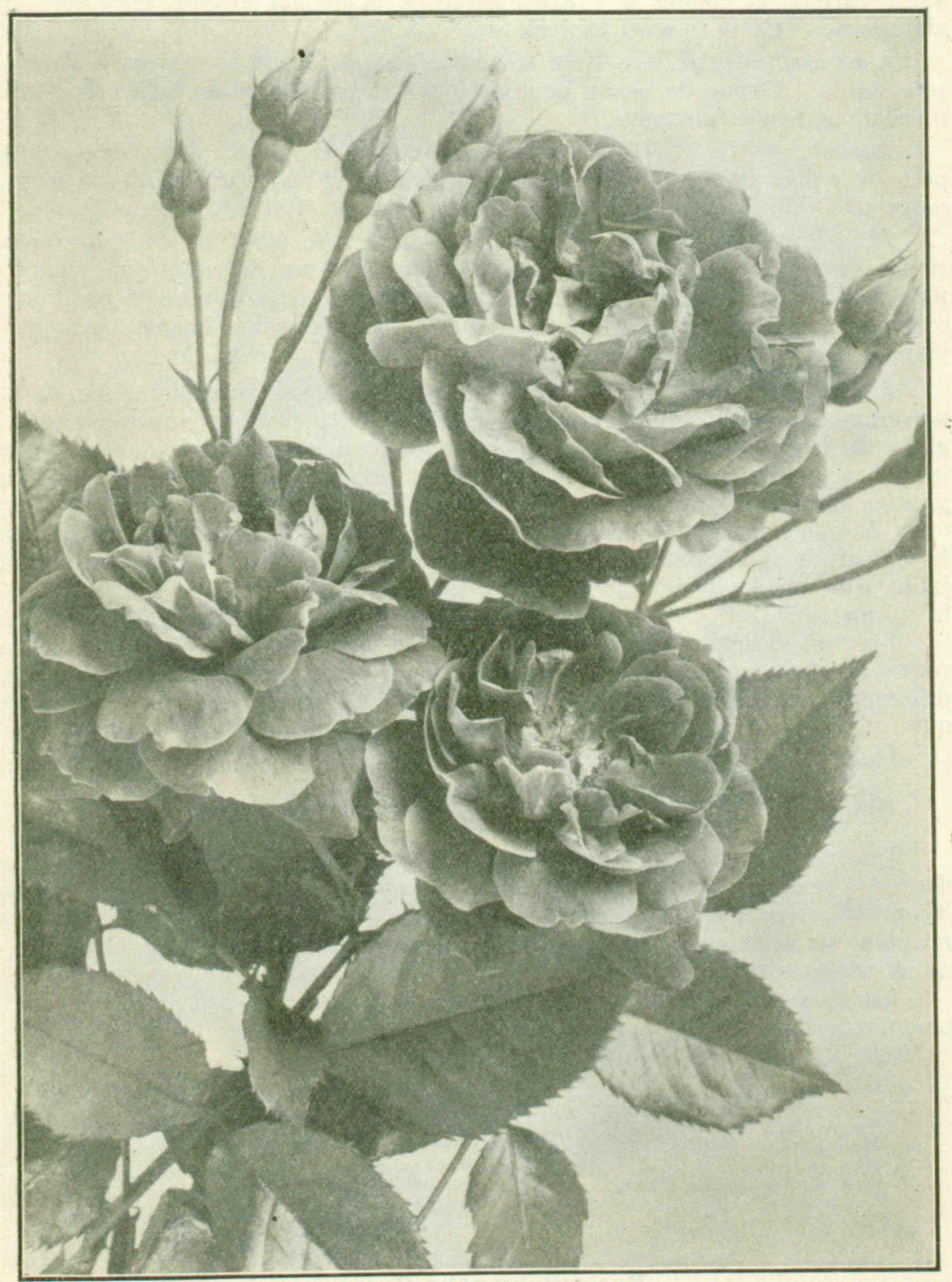

Gruss an Teplitz-Une rose hybride le thé très vigoureuse et à floraison abondante. 
tres variétés, elles ont beaucoup de bonnes qualités, notamment la floraison, qui est assez abondante et persistante pour en faire une des roses de jardin les meilleures et les plus satisfaisantes. De couleur elles sont rouge cramoisi, tirant sur l'érarlate, et comme il y a beaucoup de fleurs par grappe, elles sont très attrayantes. Cette rose est très odorante.

Lady Ashtown, H. T.-Rose très satisfaisante, facile à cultiver, à floraison persistante. Donne de beaux boutons longs et pointus et de belles fleurs rose foncé d'une bonne substance.

Lady Pirrie, H. T.-Très bonne rose thé hybride. L'une de celles dont l'aspect est le plus attrayant. Les fleurs sont rose cuivré avec une teinte de saumon rougeâtre. L'intérieur des pétales est jaune abricot. Cette rose est odorante.

Mme Edouard Herriot, Pernetiana.-Cette rose est l'une des plus vigoureuses de toutes les roses Pernetiana à Ottawa et l'une de celles qui produit le plus de fleurs. La couleur de la fleur a été décrite de plusieurs façons; terracotta, rouge corail, rose écarlate vif, rouge écrevisse et rouge brique, ombragé de jaune. Très belle combinaison de couleurs. La fleur est semi-double.

Madame Ravary, H. T.-Aucune liste de bonnes roses hybrides de thé ne serait complète si elle ne comptait une des belles variétés jaunes qui sont la gloire de leur eatégorie. On ne pourrait guère dire cependant que cette rose mérite sa place simplement à cause de sa couleur, car il y en a d'autres qui sont presque aussi belles, telles que les Mrs. Aaron Ward, Le Progrès, Margaret Molyneux, Joseph Hill, mais Madame Ravary a donné de bons résultats à Ottawa par la vigueur de sa végétation et l'abondance de ses fleurs. Elle a de beaux boutons jaune d'or qui deviennent jaune orange en s'ouvrant; c'est une belle rose pour la culture en ville.

Les sept variétés de roses thé hybrides qui viennent d'être décrites comptent parmi les meilleures de leur catégorie pour un petit jardin. Les roses sont de différentes couleurs. La durée de leur saison de floraison est très longue, plus que chez les hybrides remontantes.

\section{Roses Pernetianas}

Une nouvelle race de roses appelée "Pernetiana" a été introduite en ces dernières années. Elles sont nommées d'après M. Pernet-Ducher, le fameux hybridiste et producteur français de roses, qui a donné au monde les premiers membres de cette catégorie et beaucoup d'autres. Elles sont très appréciées à cause de leur superbe couleur, spécialement dans les teintes oranges et jaunes.

Les roses qui suivent comptent parmi les meilleures et celles qui donnent le plus d'espoir:-

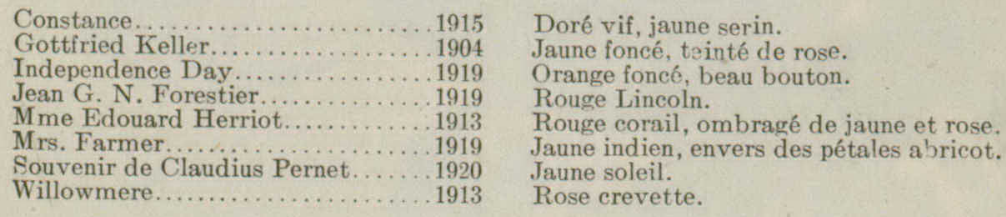

\section{Roses Polyantha Pompons}

Quelques-unes des meilleures acquisitions à la liste des roses rentrent dans la catégorie des Polyantha Pompons. Ces variétés naines donnent des fleurs continuêflement à partir du commencement de la saison des roses jusqu'à la fin de l'automne. Elles sont très utiles comme plantes de bordure dans la roseraie. 


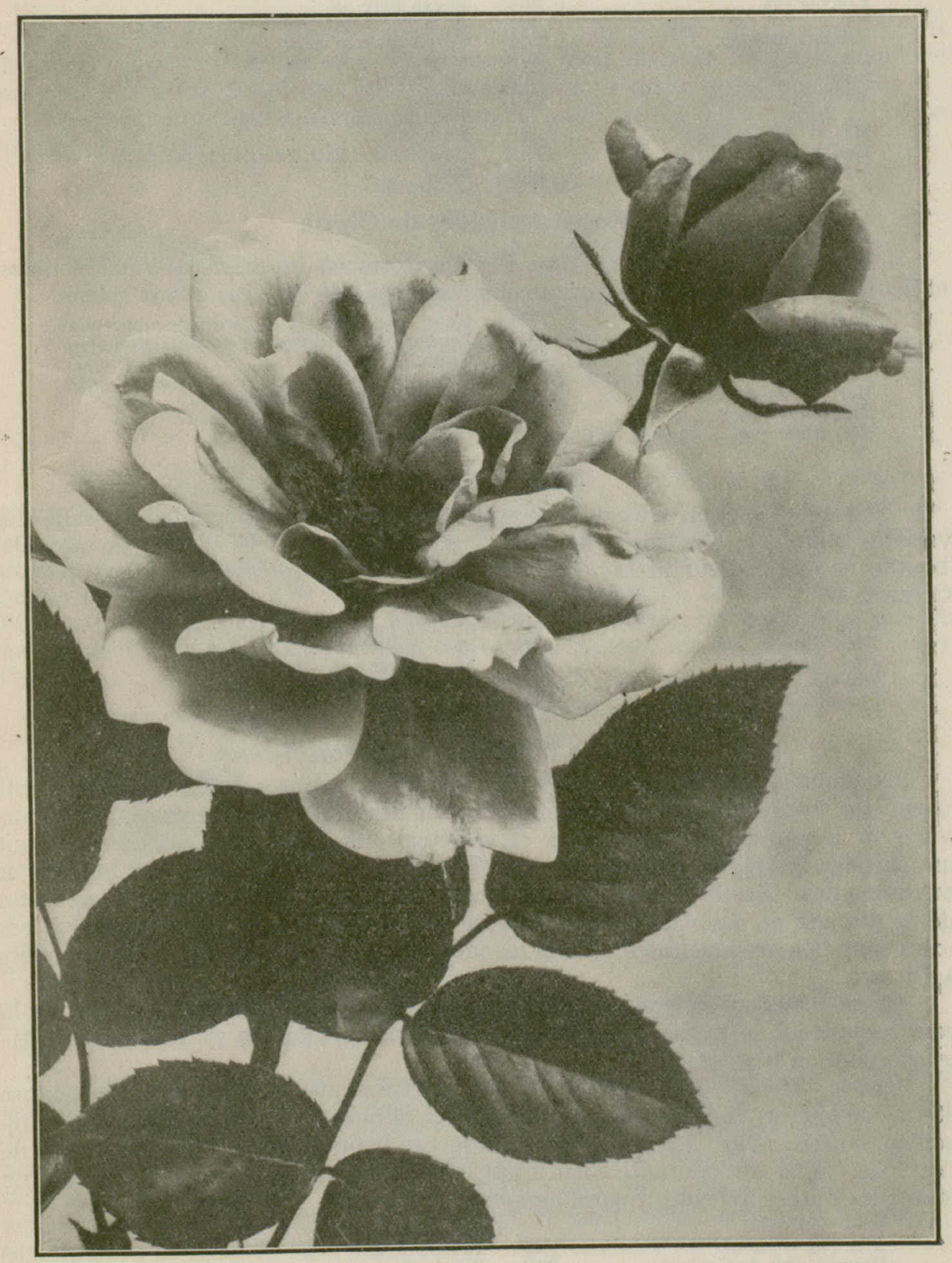

Madame Edouard Herriot-Une des meilleures des roses Pernetianas 
Il est facile de les protéger contre l'hiver à cause de leur pousse naine et compacte. Elles sont donc spécialement désirables.

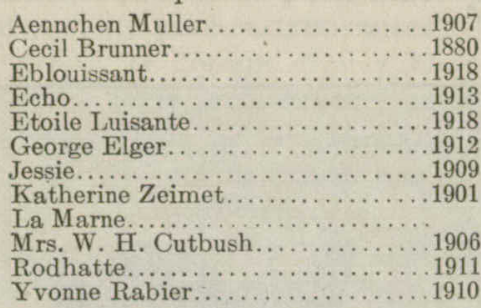

Rose vif.

Blane, ombragé de rose pâle.

Rouge foncé vif.

Rose pâle, semi-double, grosses fleurs.

Carmin et rose corail.

Jaune.

Rouge vif.

Blane.

Rose saumon, semi-double.

Rose pâle.

Rouge cerise clair, fleurs simples, grosses.

Blane.

\section{Roses hybrides de Chine}

Ces roses à l'exception de Mme Plantier forment des arbustes à pousse basse et fleurissent sans arrêt à partir du commencement de l'été jusqu'aux gelées.

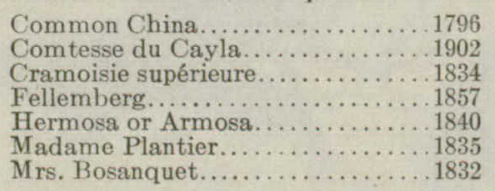

Rose, rose mensuelle du jardin de grand'mère.

Jaune cuivré, ombragé de carmin, odorante.

Cramoisi velouté.

Rose cramoisi.

Rose pâle, floraison abondante.

Blane, forme de gros arbrisseaux.

Couleur chair délicate.

\section{Roses simples}

Une autre section très intéresssante et relativement nouvelle est celle des variétés simples. Ces roses fleurissent abondamment. Elles ont un superbe bouton et une couleur attrayante. Les variétés suivantes ont été éprouvées à Ottawa:-

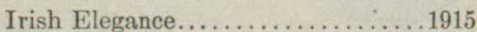

Irish Glory ..................... 1900

Irish Harmony................... 1904

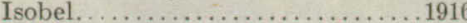

Simplicity.................... 1909
Abricot, boutons écarlate orange.

Rose argenté et cramoisi.

Jaune safran et écarlate.

Saumon carmin, base jaunâtre.

Blane pur.

\section{Roses hybrides du Japon}

Quelques-unes des variétés les plus rustiques de roses cultivées se trouvent parmi les roses hybrides du Japon. Ce sont des croisements entre la rose sauvage du Japon et d'autres espèces et variétés. La plupart de ces hybrides ont un feuillage lustré, de belle apparence. Elles sont très résistantes aux insectes et aux maladies. Les fleurs sont généralement simples ou semi-doubles. Les fruits de la plupart de ces variétés sont gros et de belle apparence, de sorte que ces roses sont très ornementales. Voici les meilleures de celles qui ont été éprouvées à Ottawa.

Outre celles qui se trouvent dans la liste suivante, il y aurait à mentionner la rose Agnes, qui se trouve à la ferme expérimentale centrale et que nous multiplions pour la plantation:-

Agnes.-Rosa rugosa x Jaune de Perse. Cette plante, par sa pousse, la texture et la couleur de ses feuilles, ressemble à $R$. Rugosa. La fleur est double, de couleur ambre pâle. La forme du bouton est bonne mais la fleur épanouie ne l'est pas. Elle est odorante, fleurit tôt. Elle se distingue sous ce rapport de toutes les autres hybrides rugosa essayées.

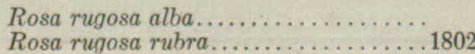

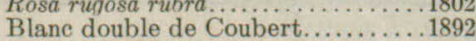

Conrad F. Meyer................. 1900

F. J. Grootendorst.............. 1920

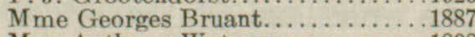

Mrs. Anthony Waterer.............1898

Rose à parfum de l'Hay............ 1904

Rose apples.................... 1906

Roseraie de l'Hay....

Souvenir de Philemon Cochet......
}

Blanc pur, simple.

Rose, simple.

Blane pur, semi-double.

Rose argenté, double.

Rouge vif, petite, double.

Blanc, semi-double.

Cramoisi foncé, semi-double.

Rouge vif, très odorante.

Rose carmin pâle.

Rouge foncé, double.

Blanc pur, double. 


\section{ROSES DIVERSES - RUSTIQUES À OTTAWA}

\section{Roses mousse}

La plupart des variétés de ces roses hâtives populaires ont été éprouvées à Ottawa. Beaucoup d'entre elles sont rustiques mais nous ne les recommandons pas pour les petites collections, car il y a beaucoup d'autres roses plus belles.

Les meilleures variétés sont les Blanche perpétuelle, Vieux rose, Blanche Moreau et Crested.

\section{Roses de Damas}

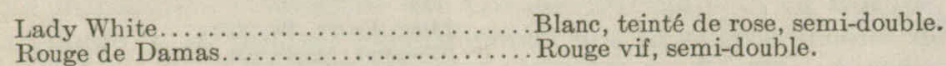

\section{Églantines d'Autriche}

Cuivrée d'Autriche.................. Rouge cuivré vif, envers des pétales vieil or, simple.

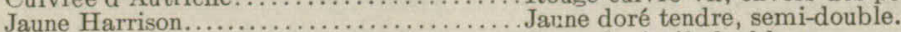

Jaune de Perse............................... doré vif, double.

\section{Roses fleurissant tard en automne}

Le mois de juin est le grand "mois des roses". Mais on sera heureux de savoir qu'il existe des roses en nombre toujours croissant qui donnent une deuxième récolte de fleurs vers la fin de l'été et en automne. En fait, certaines variétés continueraient à fleurir jusqu'en décembre, (comme elles le font en Colombie-Britannique et dans les parties sud de l'Angleterre), si elles n'étaient fauchées par les fortes gelées de la fin d'octobre. A Ottawa, les variétés nommées dans la liste suivante donnent, depuis plusieurs années, un bel étalage de fleurs d'automne.

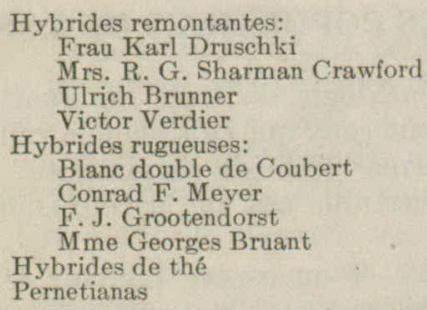

\section{ROSES REMONTANTES-LISTE DES VARIÉTÉS LES PLUS RUSTIQUES ET LES MEILLEURES}

Il y aurait grand besoin de roses remontantes plus rustiques pour les parties les plus froides du Canada et dans les travaux de sélection en cours au service de l'horticulture nous avons combiné les variétés remontantes les plus rustiques avec les espèces sauvages très rustiques, dans l'espoir d'obtenir des espèces remontantes plus rustiques. En attendant nous donnons la liste des variétés suivantes, qui ont donné satisfaction à Ottawa, et qui pourrait être utile à ceux qui se proposent de planter cette espèce de roses:

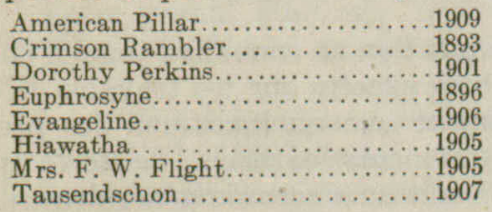

Rose, grosse, simple.

Cramoisi vif, double.

Rose écaille, petite, double.

Rose éclaboussé, petite, semi-double.

Blanc, bords roses, grosse, simple, odorante.

Cramoisi foncé, simple.

Rose, centre blane, semi-double.

Rose, rose teinté, grosse, semi-double. 
A la fin du dix-huitième siècle la rose de Chine ( $R$. indica), à floraison persistante, fut apportée en Europe. Avant longtemps il se produisit des hybrides de cette rose avec $R$. gallica et Provence. Ces hybrides furent appelés "Hybrides de Chine", mais ils descendaient de leurs parents européens, en ce sens qu'ils ne fleurissaient qu'une fois dans la saison. Ces hybrides furent ensuite croisés avec $R$. damascena et ce croisement donna naissance à la rose du Roi, une remontante de Damas qui fut introduite en 1819. Cette rose fut appelée "perpétuelle " ou "remontante" parce qu'elle avait une deuxième saison de floraison. Six ans plus tard la "Gloire de Rosamenes" apparut et l'amélioration de cette catégorie fut rapide. Les favorites du jardin comme Général Jacqueminot et Jules Margotten furent introduites vers 1819. En Grande-Bretagne et en Europe, où la saison est plus longue, il se produit généralement une deuxième récolte de fleurs, mais au Canada beaucoup des hybrides remontantes ne fleurissent qu'une fois.

La Rose de Chine à odeur de thé $(R$. indica odorata) a été croisée avec des hybrides perpétuelles, et les roses thé hybrides sont sorties de ce croisement.

Roses hybrides de thé.-Les caractères qui distinguent les hybrides de thé des hybrides remontantes sont les suivants: en ce qui concerne les plantes, une pousse plus hâtive, (et c'est pour cela qu'ils sont à peine moins rustiques que les hybrides remontantes). En ce qui concerne les fleurs, une période de floraison plus longue au printemps et à l'automne, une pétale plus profonde, des fleurs plus grosses, des couleurs plus variées et souvent une floraison plus abondante.

La rose La France (1867), une rose remarquable, a, pendant quelque temps, été classée comme hybride remontante, et n'a été mise dans sa famille actuelle qu'en 1873, lorsque la rose hybride Cheshunt fut introduite, comme première rose d'une nouvelle race, par Messieurs Paul et Fils. On peut done dire que la nouvelle race des hybrides de thé n'est venue en existence que vers 1870 , et ce n'est que lorsque la rose Caroline Testout a été introduite en 1890 que des roses de cette catégorie commencèrent à se disputer la faveur publique avec les hybrides remontantes. A partir de cette année-là les progrès ont été rapides et sûrs, et aujourd'hui cette catégorie renferme beaucoup plus de roses belles et sûres que toutes les autres catégories combinées. Ces progrès peuvent être indiqués par les chiffres suivants: en 1890, il n'y avait que six roses hybrides de thé; en 1892 , il y en avait 12 , en 1894,31 , et en 1901 il y en avait 65 ; aujourd'hui il y en a plusieurs centaines de cette catégorie.

Origine de la nouvelle race de Pernetianas.-La Jaune de Perse, une rose d'une merveilleuse couleur jaune d'or, est l'un des parents de la nouvelle race de roses qui attire aujourd'hui, et à juste titre, beaucoup d'attention. Cette rose a été créée par feu Sir H. Willcock en 1838. Elle présente un beau coup d'œil lorsqu'elle est en fleurs. Cependant, elle ne fleurit qu'une fois au commencement de l'été. Les fleurs sont petites et ne conviennent pas pour la coupe,- ces raisons expliquent pourquoi cette rose n'a pas été plus recherchée. Depuis qu'elle a été introduite, pendant toutes ces années d'existence, elle a acquis des droits à notre respect en devenant la mère par excellence d'une nouvelle rose. Pourquoi? parce qu'elle transmet à sa progéniture toutes les vertus de la jaune de Perse et pas un seul de ses défauts. Comme l'a dit M. Dickson: "Est-ce que M. Pernet-Ducher, lorsqu'il a croisé Antoine Ducher et la Jaune de Perse, avec toute son habileté et son expérience, a jamais prévu qu'il obtiendrait une fleur aussi belle que la Rose de Lyon ou le Rayon d'or?"

L'autre parent était une rose appelée Antoine Ducher. Elle n'existe plus aujourd'hui. Citons ce que l'on a dit en d'autres endroits au sujet de ce croisement:

"Il y a quelque quinze années, dans un jardin qui n'est pas loin des rives de la Méditerranée, on présentait à la Jaune de Perse une épouse d'un teint supérieur à la moyenne en la personne d'Antoine Ducher. Quels espoirs ces roses de semis ont dû faire naître dans le cœur de l'hybridiste! 
Avec quel plaisir il a présenté le premier enfant de cette union, "Soleil d'or", en 1900. C'était une rose qui devait devenir historique. Elle était vêtue d'un feuillage vert brillant, dont le parfum d'ananas charmait tout le monde. Les fleurs orange, rouge et jaune sont assez plates et n'ont pas une très belle forme, mais cependant elles sont charmantes. On recherchait beaucoup les roses d'exposition dans ces jours et comme il ne convenait pas dans ce but, le Soleil d'or, ne fit pas une grande sensation; il n'intéressa qu'un petit nombre de personnes. C'était cependant la première rose d'une nouvelle race sur laquelle l'amateur se mit tranquillement au travail, et les années se passèrent.

"Six ans plus tard on apprit en Angleterre que la plus belle rose que l'on avait jamais vue venait d'être introduite au pays. Qui oubliera la stupéfaction causée par la première apparition de cette rose parmi les hybridistes anglais à cette réunion d'avril de la société royale d'horticulture! Comme elle a encore charmé les visiteurs à l'exposition francobritannique en 1908! Ainsi, de même que son ancêtre, la rose de Lyon, l'orgueil de la race, la progéniture de Mme Mélanie Soupert et du Soleil d'or, s'est répandue dans les jardins de tous les pays."

NOTES SUR LES PARENTS DE QUELQUES ROSIERS MODERNES

\begin{tabular}{|c|c|c|}
\hline & $\begin{array}{c}\text { Pays } \\
\text { d'origine }\end{array}$ & $\begin{array}{l}\text { Epoque de } \\
\text { l'introduction }\end{array}$ \\
\hline 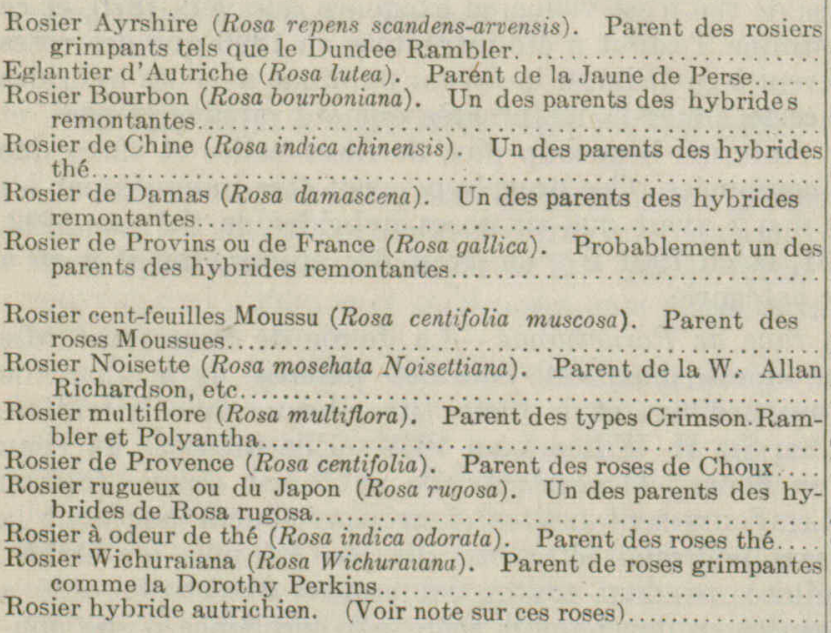 & 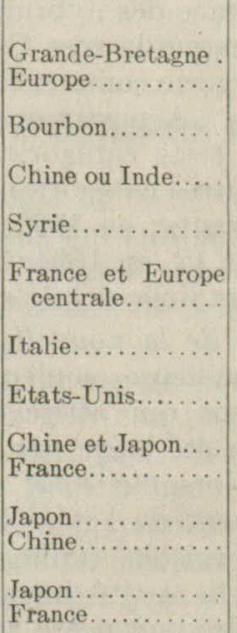 & $\begin{array}{c}\text { Inconnu } \\
1586 \\
1825 \\
1770 \\
1573 \\
\text { Inconnu } \\
1735 \\
1817 \\
1781 \\
1596 \\
1784 \\
1810-24 \\
1887 \\
1900\end{array}$ \\
\hline
\end{tabular}

\section{Bonnes roses pour les régions de la côte de la Colombie-Britannique}

Les régions de la côte de la Colombie-Britannique ont un elimat si doux, si différent de celui des autres parties du Canada que les roses qui ne sont pas rustiques ailleurs, réussissent bien cependant près de la côte ouest de cette province. La liste suivante comprend les roses qui ont donné de bons résultats à la ferme expérimentale d'Agassiz, C.-B., et à la station expérimentale de Sidney, C.-B., et ohez des producteurs particuliers. On y trouvera quelques-unes des variétés les plus belles et les plus sûres qui existent. Il y a sans doute tant de variétés de roses qui réussissent bien dans la région de la côte de la Colombie- 
Britannique qu'une liste aussi courte ne contient relativement qu'un petit nombre des bonnes variétés aptes à la plantation. Les roses les plus appréciées viennent en premier lieu sur ces listes.
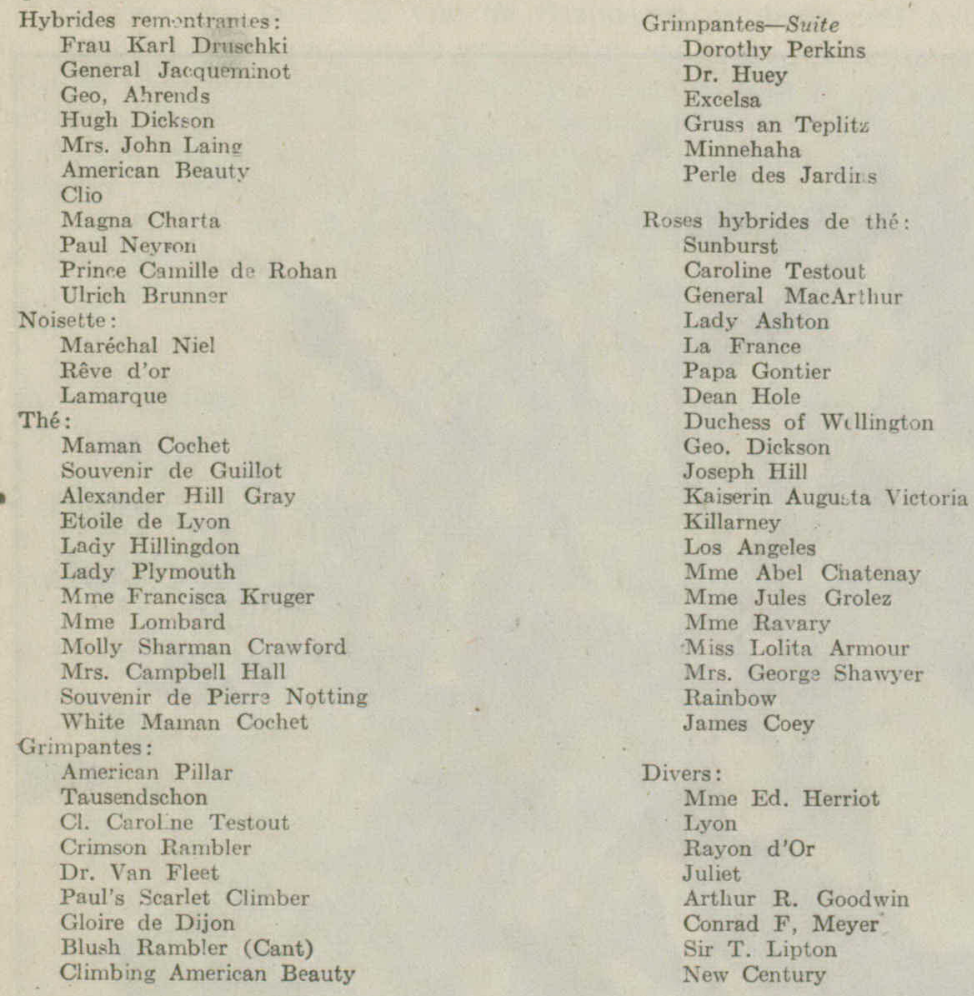

\section{La rose idéale de jardin}

Vers la fin du siècle dernier, la rose idéale appartenait à un type cultivé pour fins d'exposition. Aujourd'hui la rose de jardin est le type favori. Autrefois, on cultivait principalement la rose en vue des expositions. Ce fait nous aide à comprendre la différence qui existe entre ces deux types. Aujourd'hui on demande une rose qui réponde aux deux objets si cela est nécessaire, mais qui réussisse également comme rose de jardin. L'exposant se servait des mêmes points que ceux dont on se sert aujourd'hui en appréciant ces roses, mais il les classait dans l'ordre suivant: (1) forme, (2) couleur, (3) floraison, (4) parfum, (5) constitution.

Aujourd'hui, dans les bonnes roseraies, la constitution vient en premier lieu. Nous énumérons ci-dessous les points que l'on considère en estimant une rose.

1. Constitution.-Une plante doit être d'une vigoureuse constitution, pour produire un grand nombre de fleurs de bonne qualité. Elle doit, pour la même raison, ne pas être attaquée par les insectes et les maladies fongueuses.

2. Floraison.-La plante doit avoir une floraison perpétuelle, c'est-à-dire produire une récolte de fleurs au printemps et une autre en automne et par intermittence en été. Il est préférable que ces fleurs soient produites séparément sur de fortes tiges.

3. Couleur.-La couleur est principalement une affaire de goût personnel. Mais la couleur d'une rose idéale doit durer pendant quelques jours sans se faner. Elle doit être riche et attrayante, plutôt que terne. 


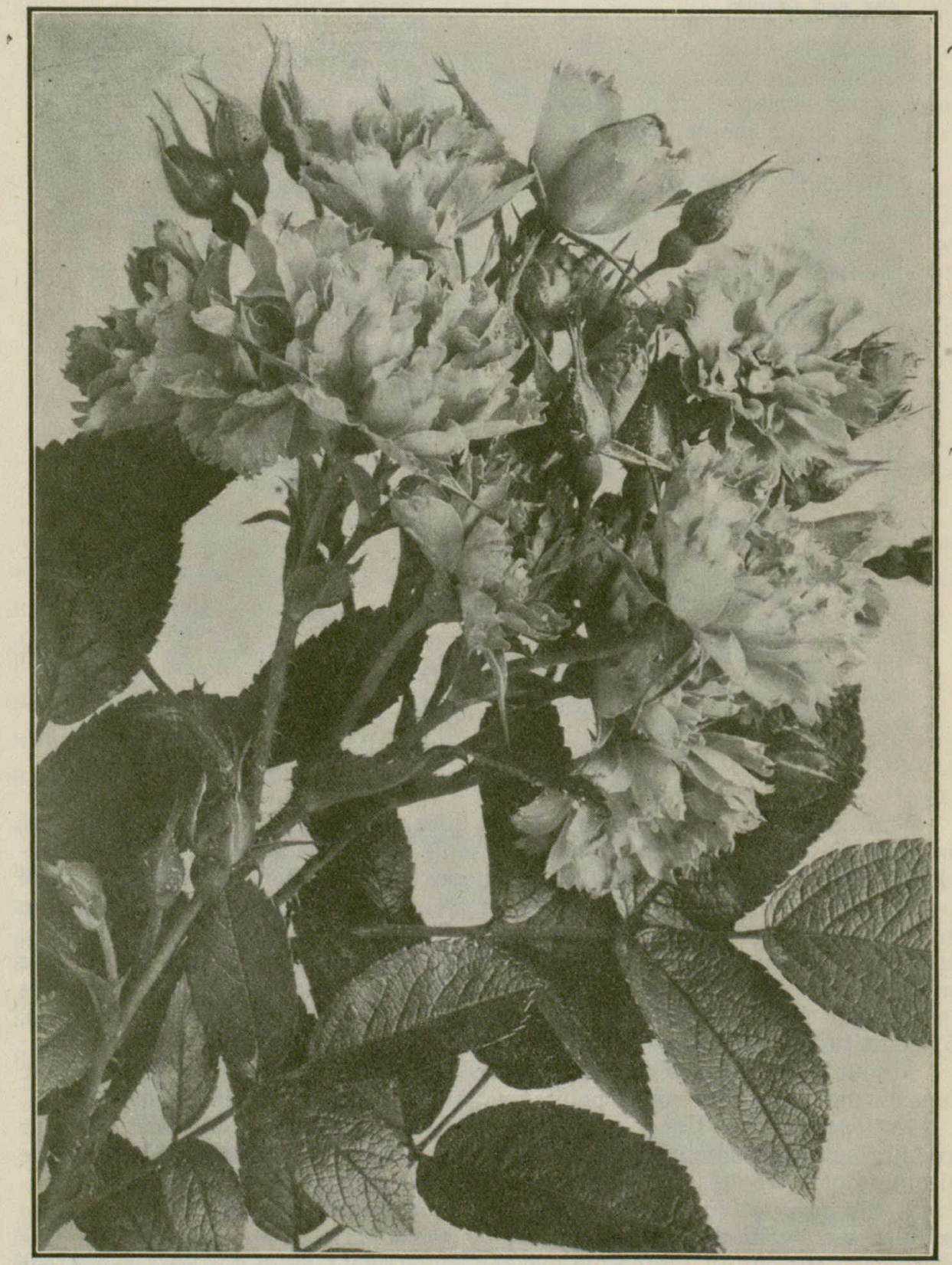

F. J. Grootendorst-Une excellente hybride Rugosa. 
4. Parfum.-Le parfum est la moitié du charme d'une roseraie. Son importance est secondaire au point de vue de l'exposant, mais elle augmente beaucoup la valeur de la rose au point de vue du jardinier.

5. Forme.-Au point de vue de l'exposant, la fleur doit avoir une forme idéale, mais au point de vue du producteur ordinaire, la forme n'est pas aussi importante. Quelques-unes des meilleures roses ne sont pas aussi bonnes sous ce rapport que l'on pourrait le désirer. Généralement parlant, la fleur à forme parfaite est celle qui est longue et en pointe avec les pétales suffisamment détachées les unes des autres pour donner du caractère et du charme à la fleur. Les pétales doivent également être de bonne substance pour qu'ils puissent conserver leur forme plusieurs jours.

\section{Roses rustiques essayées à Ottawa}

Nous avons un jardin de roses à la ferme expérimentale centrale depuis 1891, et nous y avons essayé un grand nombre de variétés en ces 132 dernières années. Avant d'enlever la vieille plantation à l'automne de 1911, nous avons pris des notes sur les variétés qui s'étaient montrées les plus rustiques, notes que nous reproduisons avec d'autres dans le tableau suivant. Pour définir le degré et la qualité du parfum de ces roses nous nous sommes servis de la marque $\mathrm{x}$. Trois $\mathrm{x}$ représentent le plus haut degré de parfum et un $\mathrm{x}$ le degré le plus bas, quand la rose est odorante. Le rang indique la qualité relative générale de la variété.

\begin{tabular}{|c|c|c|c|c|c|}
\hline Nom & Planté & Rang & Couleur & 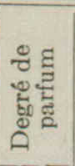 & Remarques \\
\hline 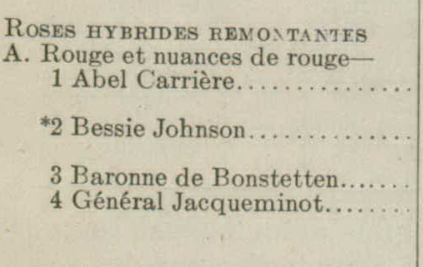 & $\begin{array}{l}1896 \\
1894 \\
1897\end{array}$ & 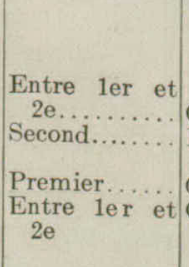 & $\begin{array}{l}\text { Cramoisi-pourpre. } \\
\text { Rouge tournantsur } \\
\text { le mauve. } \\
\text { Cramoisi-noirâtre. } \\
\text { Cramoisi-écarlate. }\end{array}$ & $\begin{array}{r}\mathrm{XXX} \\
\mathrm{XX}\end{array}$ & $\begin{array}{l}\text { Arbuste vigoureux } \\
\text { Arbuste modéré- } \\
\text { ment vigoureux. }\end{array}$ \\
\hline 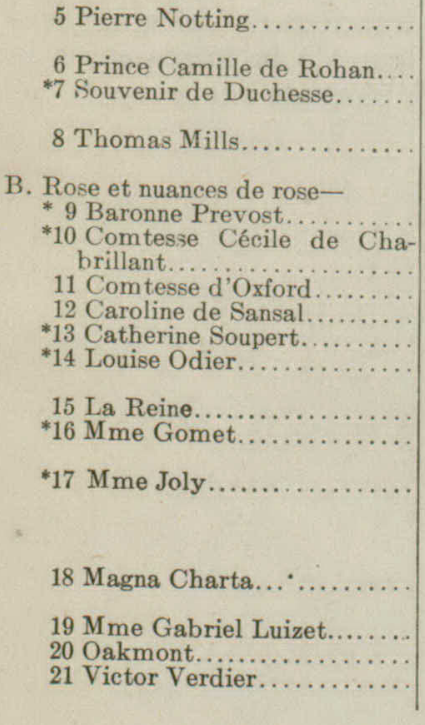 & $\begin{array}{l}1895 \\
1894 \\
1894 \\
1894 \\
1899 \\
1895 \\
1901 \\
1896 \\
1894\end{array}$ & 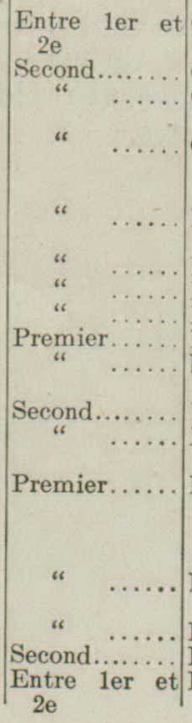 & 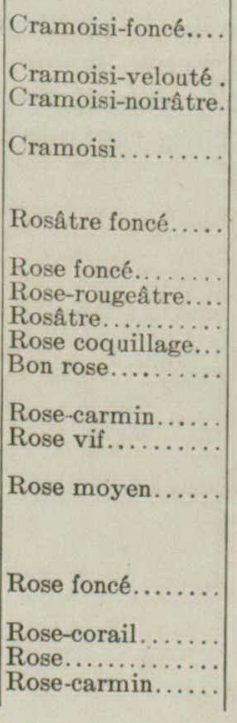 & $\begin{array}{r}\mathrm{Xx} \\
\mathrm{xx} \\
\mathrm{xxx} \\
\mathrm{XX} \\
\mathrm{Xx} \\
\mathrm{x}\end{array}$ & $\begin{array}{l}\text { Floraison très } \\
\text { abondante. } \\
\text { Type très ouvert. } \\
\text { Floraison très } \\
\text { abondante. } \\
\text { Arbuste vigoureux } \\
\text { longue saison; } \\
\text { fleurs de bonne } \\
\text { forme. } \\
\text { Floraison abon- } \\
\text { bondante. } \\
\text { Saison longue. } \\
\text { Bonne floraison. }\end{array}$ \\
\hline
\end{tabular}




\begin{tabular}{|c|c|c|c|c|c|}
\hline Nom & Planté & Rang & Couleur & 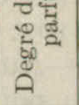 & Remarques \\
\hline Roses DIVERses & & & & & \\
\hline 22 Mme Plantier (blane)........ & 1897 & Premier..... & Blanc.......... & $\mathrm{x}$ & $\begin{array}{l}\text { Vigoureux; florai- } \\
\text { son abondante; } \\
\text { une des meil- } \\
\text { leures roses blan- } \\
\text { ches, }\end{array}$ \\
\hline $\begin{array}{r}\text { Roses moussues }- \\
23 \text { Blanche Moreau............ }\end{array}$ & 1894 & Premier... & Blanc....... & $\mathrm{xx}$ & $\begin{array}{l}\text { Floraison abon- } \\
\text { dante; très vi- } \\
\text { goureux. }\end{array}$ \\
\hline $\begin{array}{l}24 \text { Roses moussues blanches re- } \\
\text { montantes................ } \\
25 \text { Centifolia............... }\end{array}$ & $\begin{array}{l}1896 \\
1894\end{array}$ & 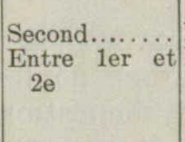 & $\begin{array}{l}\text { Blanc } \ldots \ldots \ldots \ldots \\
\text { Blanc.............. }\end{array}$ & $\begin{array}{l}x x \\
x x\end{array}$ & $\begin{array}{l}\text { Floraison abon- } \\
\text { dante; très vi- } \\
\text { goureux. }\end{array}$ \\
\hline & 1896 & Premier...... & $\begin{array}{l}\text { Rouge tirant sur } \\
\text { mauve. }\end{array}$ & $\mathrm{xx}$ & $\begin{array}{l}\text { Floraison abon- } \\
\text { dante. }\end{array}$ \\
\hline $\begin{array}{l}27 \text { Autres roses moussues } \\
\text { nommées. }\end{array}$ & 1894 & Second....... & $\begin{array}{l}\text { Nuances de rose et } \\
\text { rouge. }\end{array}$ & $x x$ & Tous vigoureux. \\
\hline $\begin{array}{l}\text { Roses rugueuses- } \\
28 \text { Rugueuses blanche et rouge }\end{array}$ & $1897 \mathrm{et}$ & & & & imples et. \\
\hline & plus tard & Premier. & $\begin{array}{l}\text { de rose et de } \\
\text { mauve. }\end{array}$ & & roses doubles. \\
\hline $\begin{array}{l}\text { Roses de Damas- } \\
29 \mathrm{Mme} \text { Hardy (blane)......... }\end{array}$ & 1894 & Second.... & $\begin{array}{l}\text { Blane tirant sur le } \\
\text { rose. }\end{array}$ & $\mathbf{x}$ & Vigoureux. \\
\hline $\begin{array}{l}\text { Rugueuses hybrides- } \\
30 \mathrm{Mme} \text { Geo. Bruant........... } \\
31 \mathrm{Mme} \text { Chas. Worth......... } \\
32 \text { Agnes Emily Carman...... }\end{array}$ & $\begin{array}{l}1894 \\
\text { vers } 1897 \\
1894\end{array}$ & 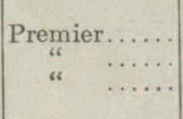 & 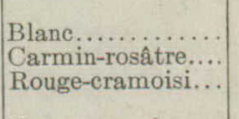 & $\begin{array}{r}x x \\
x \\
x\end{array}$ & $\begin{array}{l}\text { Vigoureux. } \\
\text { Vigoureux. } \\
\text { Floraison très } \\
\text { abondante. }\end{array}$ \\
\hline 33 Mary Arnott & $\begin{array}{l}\text { vers } \\
1897\end{array}$ & ... & Rouge-earmin... & $\mathrm{x}$ & $\begin{array}{l}\text { Floraison excep- } \\
\text { tionnellement a- } \\
\text { bondante; fleurs } \\
\text { en belles grappes }\end{array}$ \\
\hline
\end{tabular}

L'astérisque indique que ces roses ne se trouvent plus actuellement dans la plupart des pépinières. Dans bien des cas elles ont été remplacées par des roses de meilleure couleur ou de meilleure forme. Les roses hybrides de thé qui, généralement, sont supérieures au point de vue de la couleur, de la forme et de la durée de la floraison, prennent également rapidement la place de beaucoup de ces anciennes roses hybrides remontantes. 

